\title{
Scaling Laws and Dynamics of Bubble Coalescence
}

\author{
Christopher R. Anthony, ${ }^{1, *}$ Pritish M. Kamat, ${ }^{1}$ Sumeet S. Thete, ${ }^{1}$ James \\ P. Munro, ${ }^{2}$ John R. Lister, ${ }^{2}$ Michael T. Harris, ${ }^{1}$ and Osman A. Basaran ${ }^{1, \dagger}$ \\ ${ }^{1}$ School of Chemical Engineering, Purdue University, \\ 480 Stadium Mall Drive, West Lafayette, IN 47907 \\ ${ }^{2}$ Institute of Theoretical Geophysics, Department of Applied Mathematics and Theoretical Physics, \\ CMS, Wilberforce Road, Cambridge CB3 0WA, UK
}

(Dated: August 19, 2017)

\begin{abstract}
The coalescence of bubbles and drops plays a central role in nature and industry. During coalescence, two bubbles or drops touch and merge into one as the neck connecting them grows from microscopic to macroscopic scales. The hydrodynamic singularity that arises when two bubbles or drops have just touched and the flows that ensue have been studied thoroughly when two drops coalesce in a dynamically passive outer fluid. In this paper, the coalescence of two identical and initially spherical bubbles, which are idealized as voids, that are surrounded by an incompressible Newtonian liquid is analyzed by numerical simulation. This problem has recently been studied (a) experimentally using high-speed imaging and (b) by asymptotic analysis in which the dynamics is analyzed by determining the growth of a hole in the thin liquid sheet separating the two bubbles. In the latter, advantage is taken of the fact that the flow in the thin sheet of non-constant thickness is governed by a set of one-dimensional, radial extensional flow equations. While these studies agree on the power law scaling of the variation of the minimum neck radius with time, they disagree with respect to the numerical value of the prefactors in the scaling laws. In order to reconcile these differences and also provide insights into the dynamics that are difficult to probe by either of the aforementioned approaches, simulations are used to access both earlier times than it has been possible in the experiments and also later times when asymptotic analysis is no longer applicable. Early times and extremely small length scales are attained in the new simulations through the use of a truncated domain approach. Furthermore, it is shown by direct numerical simulations in which the flow within the bubbles is also determined along with the flow exterior to them that idealizing the bubbles as passive voids has virtually no effect on the scaling laws relating minimum neck radius and time.
\end{abstract}

\section{INTRODUCTION}

The phenomenon of collision and coalescence of bubbles plays a central role in a number of natural settings and industrial applications. Bubble collision and coalescence can alter the size distribution of bubbles, which can have important ramifications for the underlying phenomena. A well-known example is provided from nature where bubbles, produced from breaking ocean waves, inject salt nuclei into the atmosphere, a process that can affect subsequent raindrop production [1]. In the industrial process of microflotation, which is a technique that is widely used in wastewater treatment, small bubbles are introduced into a liquid to filter out algae, bacteria, and other waste products into a foam [2]. Recently, a variant of this technique has also been sought after as an economic way to harvest and concentrate algae grown in bioreactors for use in the production of biodiesel [3]. Bubble size distributions, which are affected by both breakup and coalescence, also play a major role in the transport and fate of gas and/or oil released during deepwater gas and/or oil spills [4]. Therefore, developing a better understanding of the coalescence of a pair of initially spher-

\footnotetext{
*anthonc@purdue.edu

† obasaran@purdue.edu
}

ical bubbles, which is the subject of this paper, is desirable both in studies of natural phenomena and for the improvement of existing and/or development of new technological applications.

When two bubbles or drops are gently brought together and touch, a microscopic fluid bridge forms between them. At the instant of contact, the surface tension or capillary pressure is singular because of the infinite curvature of the fluid interface at the point of contact. The dynamics as the bridge grows and the two bubbles or drops merge into one are driven by this initial singularity in capillary pressure. The coalescence singularity has been studied exhaustively when two drops coalesce in a dynamically passive outer fluid, e.g. air or vacuum [5-18]. Paulsen et al. [15] have presented a phase diagram of coalescence in such situations in the parameter space comprised of a dimensionless ratio of the governing forces (viscous force divided by the square root of the product of inertial and surface tension forces) and the instantaneous radius of the bridge connecting the two drops divided by the initial drop radius that (a) divides the parameter space into three regimes consisting of an initial asymptotic regime where all three forces are important and two final regimes where either viscous and surface tension forces or inertial and surface tension forces are important, and (b) identifies the values of the dimensionless bridge radius for which the dynamics will transition from the initial asymptotic regime to one or the other of 
the two final regimes. Also noteworthy is the paper by Duchemin et al. [9] in which the coalescence of two inviscid (zero viscosity) drops in a passive outer fluid (air) has been analyzed by means of boundary integral simulations. These authors have shown that the surfaces of the thin retracting sheet of air between the drops reconnect in finite time to form a toroidal enclosure. In studying drop coalescence experimentally, it is possible to explore extremely small length and time scales in the immediate aftermath of the singularity. Although such measurements cannot be performed by imaging, which is the most commonly used method for studying both coalescence and breakup, they are readily accomplished by means of electrical conductivity measurements as were first carried out by Case \& Nagel [12] and yield how the minimum radius of the fluid bridge varies with time.

While virtually all of the studies cited in the previous paragraph were carried out when the fluid outside the drops could be treated as dynamically passive, considerably fewer studies have considered the effect of a dynamically active outer fluid [19-25]. Recently, Paulsen et al. [25] experimentally investigated the effect of the external fluid on both drop and bubble coalescence. Of particular interest to the present paper is their study of the limiting case of two bubbles coalescing in a dynamically active outer fluid. However, as the bubbles in their experiments were non-conducting, Paulsen et al. were only able to study bubble coalescence via imaging and hence were unable to probe the dynamics at extremely early times and/or for small length scales. Nevertheless, Paulsen et al. showed that there are two distinct regimes of bubble coalescence depending on the viscosity of the exterior liquid. For low-viscosity outer liquids, the dynamics fell in an inertial regime. For high-viscosity outer liquids, the dynamics lay in a viscous regime. In both regimes, however, the minimum neck radius varied as the square root of time. More recently, Munro et al. [26] used asymptotic analysis in which they determined the growth of a hole in the thin liquid sheet separating the two bubbles. In their analysis, these authors constructed similarity solutions to a third order system of radial extensional flow equations for the thickness of the sheet and the velocity within it. These authors thereby showed that the minimum neck radius increased as time raised to the one half power, in accord with experiments. While the power-law behavior of bridge radius with time is the same from both theory and experiment, several differences emerge from a careful scrutiny of the results. First, the prefactors in the expressions relating minimum neck radius and time obtained from theory differ from those obtained from experiments. These differences may be attributable to the inability of the imaging method to probe the period in the immediate aftermath of the instant at which the two bubbles have just touched. Moreover, whereas Paulsen et al. had surmised that the appropriate length scale in the radial direction in both regimes was the radius of the bridge, Munro et al.'s theory revealed that the radial length scale is much smaller than the radius of the bridge for outer fluids of low viscosity. Furthermore, although the theory is extremely valuable in probing the early time dynamics, it becomes less reliable as the two bubbles continue to coalesce and the retracting fluid sheet loses its slenderness. Munro et al. also carried out a limited set of computations to directly simulate the coalescence of two bubbles, and thereby put on firmer foundation some of the assumptions that are inherent to the theoretical approach.

The goal of this paper is to use detailed numerical simulations to study the coalescence of two bubbles in a viscous outer fluid and thereby accurately capture the dynamics at both early and late times to overcome the aforementioned limitations of experiments and theory. The paper is organized as follows. Section II provides the mathematical formulation of the problem solved and summarizes the numerical method used to carry out the simulations. Since the fluid bridge connecting the two bubbles at the beginning of the simulations is of small but finite size, section III presents the results of tests that have been carried out to demonstrate the insensitivity of the computed scaling predictions on the initial conditions. Section IV presents the results of extensive numerical simulations and scaling laws that are deduced from the computations. Also in this section, a novel truncated domain approach is presented that permits computational investigation of the coalescence dynamics at extremely early times compared to those possible in experiments and allows closer comparison of simulation results with theory. By the same token, it is further shown in this section that the agreement between simulations and experiments can also be improved by focusing instead on the computational results at later times, i.e. times that experiments have been able to probe. The article concludes in section $\mathrm{V}$ by summarizing the results obtained in this paper and pointing out some some future avenues for extending this work.

\section{MATHEMATICAL FORMULATION AND NUMERICAL METHOD}

\section{A. Mathematical Formulation}

The system consists of two dynamically passive bubbles, both of which are initially spheres of radius $\tilde{R}$ that are connected by a small bridge or a neck, that are immersed in an exterior or outer liquid that is an incompressible Newtonian fluid of constant density $\rho$ and constant viscosity $\mu$, as shown in Fig. 1 . The air-liquid interface separating the bubbles from the surrounding liquid has constant surface tension $\gamma$. Initially, the bubbles are stationary and the liquid surrounding them is quiescent so that the fluid velocity in the region exterior to the bubbles equals zero, viz. $\tilde{\mathbf{v}}=\mathbf{0}$ at time $\tilde{t}=0$. As in earlier computational studies, the microscopic bridge that connects the two bubbles initially is of radius $\tilde{R}_{0}$ and height $2 \tilde{Z}_{0}[9,15]$ (see Fig. 1 and below). As shown in the next 


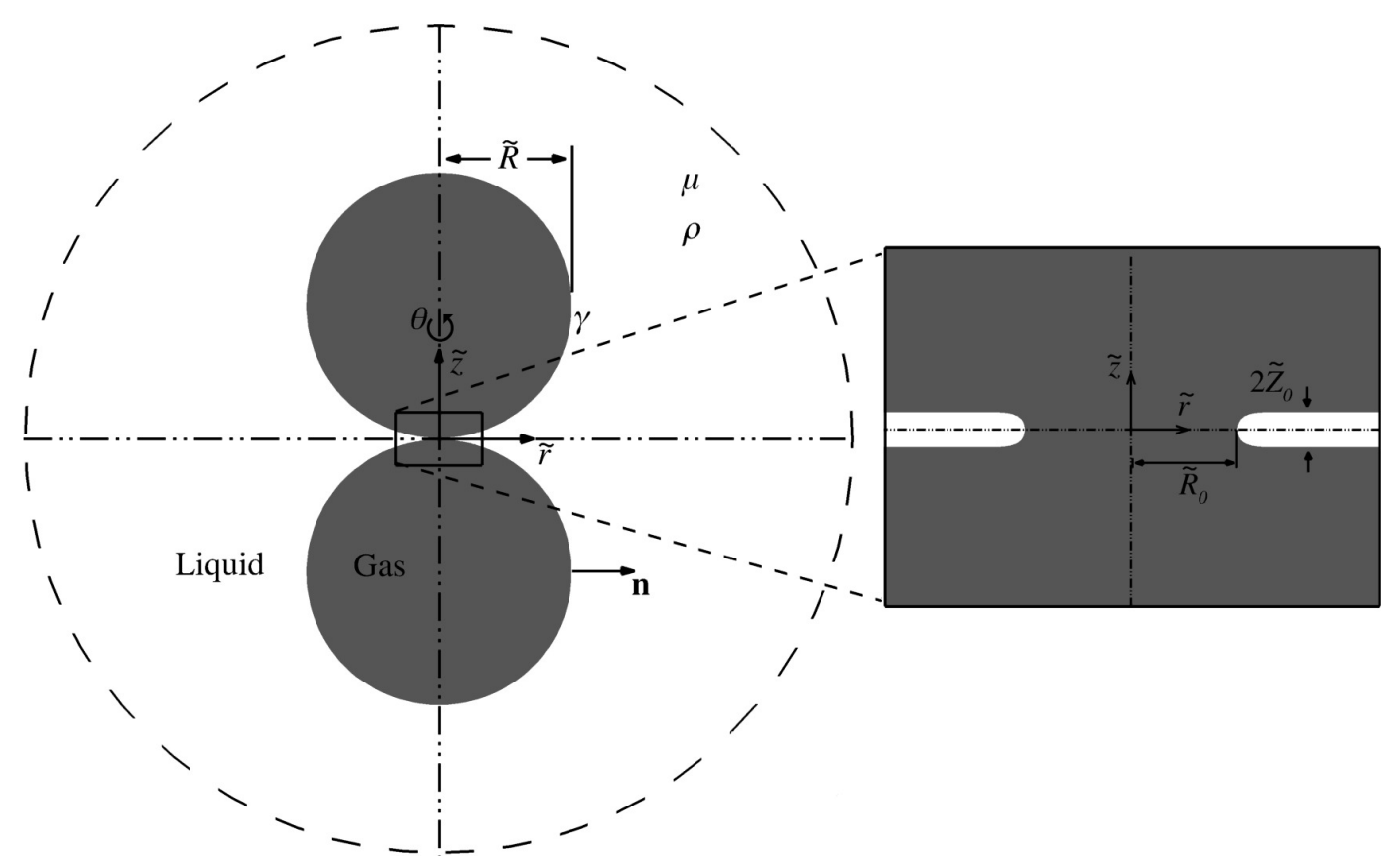

FIG. 1. The onset of the coalescence of two equal-sized bubbles in a liquid: definition sketch and initial conditions. At the initial instant, two spherical bubbles of radius $\tilde{R}$ are connected by a microscopic gas bridge of radius $\tilde{R}_{0}$ and height $2 \tilde{Z}_{0}$, a zoomed-in view of which is also shown.

section, the dimensions of this connecting bridge are chosen to be small enough such that the replacement of the actual initial condition of two spherical bubbles touching at a single point by two bubbles being connected by a small but finite-size bridge has a negligible effect on the dynamics that ensues in the aftermath of the space-time singularity that occurs at the initial instant and initiates the merging of the two bubbles into one. In what follows, it proves convenient to adopt a cylindrical coordinate system $(\tilde{r}, \theta, \tilde{z})$ with its origin at the location where the two initially spherical bubbles just touch. Thus, the $\tilde{z}$-axis runs through the centers of the two initially spherical bubbles. The dynamics is taken to be axially symmetric about the $\tilde{z}$-axis and, because the two bubbles are identical, the dynamics is also symmetric with respect to the $\tilde{z}=0$ plane. Thus, the problem domain is just one quadrant of the $\tilde{r} \tilde{z}$-plane, $\tilde{r} \geq 0$ and $\tilde{z} \geq 0$, as shown in Fig. 1. In the coordinate system that has just been introduced, the surface of the initial bridge connecting the two bubbles is given by $\left[\tilde{r}^{2}-\left(\tilde{R}_{0}+\tilde{Z}_{0}\right)\right]^{2}+\tilde{z}^{2}=\tilde{Z}_{0}^{2}$.

In this paper, the problem variables are nondimensionalized by choosing the undisturbed radii of the bubbles as characteristic length, $l_{c}=\tilde{R}$, the inertialcapillary time as characteristic time, $t_{c}=\sqrt{\rho \tilde{R}^{3} / \gamma}$, the ratio of the latter two scales as characteristic velocity, $v_{c}=l_{c} / t_{c}$, and the capillary pressure $\gamma / \tilde{R}$ as characteristic pressure/stress, $p_{c}=\gamma / \tilde{R}$. Upon the introduction of these characteristic scales, it is found that the dynamics is governed by a single dimensionless group, the Ohnesorge number $O h=\mu / \sqrt{\rho \tilde{R} \gamma}$, which is the ratio of viscous force to the square root of the product of inertial and surface tension forces. The dynamics appears to also depend on two dimensionless parameters that result from the way the initial conditions are imposed. These are the initial values of the dimensionless bridge radius and bridge height, viz. $R_{0}=\tilde{R}_{0} / \tilde{R}$ and $2 Z_{0}=2 \tilde{Z}_{0} / \tilde{R}$. Below, it is further shown that a third dimensionless parameter enters the problem during simulations. However, all three of these dimensionless parameters can be shown to have a negligible effect on the dynamics if they are judiciously chosen and once all initial transients have died down. In what follows, variables without tildes over them denote the dimensionless counterparts of those with tildes.

The dynamics in the region $\Omega(t)$ occupied by the liquid surrounding the two bubbles are governed by the NavierStokes and continuity equations which in dimensionless form are given by

$$
\begin{gathered}
\frac{\partial \boldsymbol{v}}{\partial t}+\boldsymbol{v} \cdot \boldsymbol{\nabla} \boldsymbol{v}=\boldsymbol{\nabla} \cdot \boldsymbol{T} \\
\boldsymbol{\nabla} \cdot \boldsymbol{v}=0
\end{gathered}
$$

where $\boldsymbol{v} \equiv \tilde{\boldsymbol{v}} / v_{c}$ is the dimensionless fluid velocity and $\boldsymbol{T} \equiv \tilde{\boldsymbol{T}} / p_{c}=-p \boldsymbol{I}+O h\left[\nabla \boldsymbol{v}+(\nabla \boldsymbol{v})^{T}\right]$ is the dimensionless stress tensor, with $p \equiv \tilde{p} / p_{c}$ denoting the dimensionless pressure, $t \equiv \tilde{t} / t_{c}$ is dimensionless time, and $\nabla \equiv \tilde{R} \tilde{\nabla}$ is the dimensionless gradient operator. The pressure within the bubbles is taken to be uniform in 
space and constant in time, and set to be the pressure datum.

The domain $\Omega(t)$ over which the governing Eqs. (1a) and (1b) are solved consists of the region exterior to the air-liquid interface or the free surface of the coalescing bubbles, $S(t)$, which is unknown a priori, and is bounded by the symmetry axis $r \equiv \tilde{r} / \tilde{R}=0$ and the symmetry plane $z \equiv \tilde{z} / \tilde{R}=0$, where $r$ and $z$ denote the dimensionless radial and axial coordinates in cylindrical coordinates. Because of the aforementioned axial symmetry and symmetry conditions, symmetry boundary conditions are imposed along the symmetry axis $r=0$ and the plane $z=0$. At radial distances indefinitely far from the center of mass of the two bubble system, the liquid must become quiescent. Along the free surface $S(t)$, the kinematic and traction boundary conditions are imposed to determine the unknown shape of the free surface and account for the discontinuity or jump in stress due to surface tension:

$$
\begin{gathered}
\mathbf{n} \cdot\left(\mathbf{v}-\mathbf{v}_{s}\right)=0 \\
\mathbf{n} \cdot \mathbf{T}=-2 \mathcal{H} \mathbf{n}
\end{gathered}
$$

Here, $\boldsymbol{v}_{s} \equiv \tilde{\boldsymbol{v}}_{s} / v_{c}$ is the dimensionless velocity of points on the free surface $S(t), \boldsymbol{n}$ is the outward pointing unit normal to the free surface, and $2 \mathcal{H} \equiv 2 \tilde{\mathcal{H}} \tilde{R}$ is twice the dimensionless local mean curvature of the interface.

\section{B. Numerical Method}

The aforementioned transient system of governing equations is solved numerically using a fully implicit, arbitrary Lagrangian-Eulerian (ALE), method of lines algorithm in which the Galerkin/finite element method (G/FEM) is employed for spatial discretization [27] and an adaptive, implicit finite difference method is deployed for time integration [28]. In order to capture the large deformations that the surfaces of the bubbles and hence the domain exterior to them undergo, the elliptic mesh generation method developed by Christoloudou and Scriven [29] for studying thin-film coating flows and which was later extended to simulate free surface flows of Newtonian and complex fluids with breakup and coalescence [15, 3033 ], was used to discretize the spatial domain $\Omega(t)$ and determine the radial and axial coordinates of each grid point in the moving, adaptive mesh simultaneously with the velocity and pressure unknowns in the exterior liquid and the free surface profile. The velocity and pressure unknowns were solved in the mixed interpolation sense using biquadratic basis functions to represent the velocity unknowns and bilinear basis functions to represent the pressure unknowns [34]. The locations of the mesh coordinates were also represented using biquadratic basis functions. The numerical scheme reduces the problem to a system of nonlinear algebraic equations that can be solved iteratively via a multidimensional Newton's method. The resulting system of linear equations is then solved with a multifrontal algorithm which takes advantage of the sparsity of the Jacobian matrix that arises in Newton's method. This multifrontal algorithm was inspired by the frontal method introduced by Hood [35]. For a more complete description of the numerical method that has been employed, the reader is referred to Notz and Basaran [30].

In the simulations, it is impracticable for the domain exterior to the bubbles $\Omega(t)$ to extend out to infinity. Therefore, the computational domain is cut off by a spherical surface of large but finite radius $R_{\infty}$ so that the domain consists of the region between $S(t)$ and the spherical surface located at $r=R_{\infty}$. In the simulations, it is on this sphere of large but finite radius $R_{\infty}$ rather than as $r \rightarrow \infty$ that the boundary condition that the fluid is quiescent, $\mathbf{v}=0$, is imposed. The optimal value of $R_{\infty}$ is then determined by systematically varying $R_{\infty}$ until further increases to it resulted in insignificant changes to the computed solutions.

\section{EFFECT OF BRIDGE SIZE AND INITIAL CONDITIONS ON SCALING PREDICTIONS}

As the actual initial condition of two spherical bubbles touching at a point is replaced in the simulations by two bubbles being connected by a small but finite-size bridge, in this section the effects of the initial values of the bridge radius $R_{0}$ and one half of the bridge height $Z_{0}$ on the computed predictions are examined. In particular, values of $R_{0}$ and $Z_{0}$ are varied to determine their effect on the computed variation in time of the radial and axial scales of the neck connecting the two bubbles. The time variation of the radial scale is determined by monitoring the minimum neck radius $R_{\min }(t)$, which is the instantaneous radius of the neck connecting the two bubbles at the axial location $z=0$. The axial scale $Z_{b}(t)$, or equivalently the instantaneous value of one half of the bridge height, which is hereafter referred to as the bridge half height, is determined by monitoring the variation in time of the axial location along the neck where the local value of the bridge radius equals $1.05 R_{\text {min }}$. Thus, it is expected that after the decay of initial transients, these quantities should exhibit power law responses given by $R_{\text {min }} \sim t^{\alpha_{r}}$ and $Z_{b} \sim t^{\alpha_{z}}$, where $\alpha_{r}$ and $\alpha_{z}$ are the radial and axial scaling exponents.

Figure 2(a) shows the variation of the minimum neck radius $R_{\min }$ with time in a set of simulations where the initial bridge radius $R_{0} \equiv R_{\min }(0)$ is held fixed at $10^{-3}$ but the initial bridge half height $Z_{0} \equiv Z_{b}(0)$ is varied. First, this figure makes plain that the curves depicting the variation of $R_{\text {min }}$ with $t$ for different values of $Z_{0}$ all fall on top of one another once sufficient time has elapsed. Second, the overlap between the various simulations starts earlier and earlier as the initial bridge half height $Z_{0}$ decreases. Figure 2(a) also shows that for large 

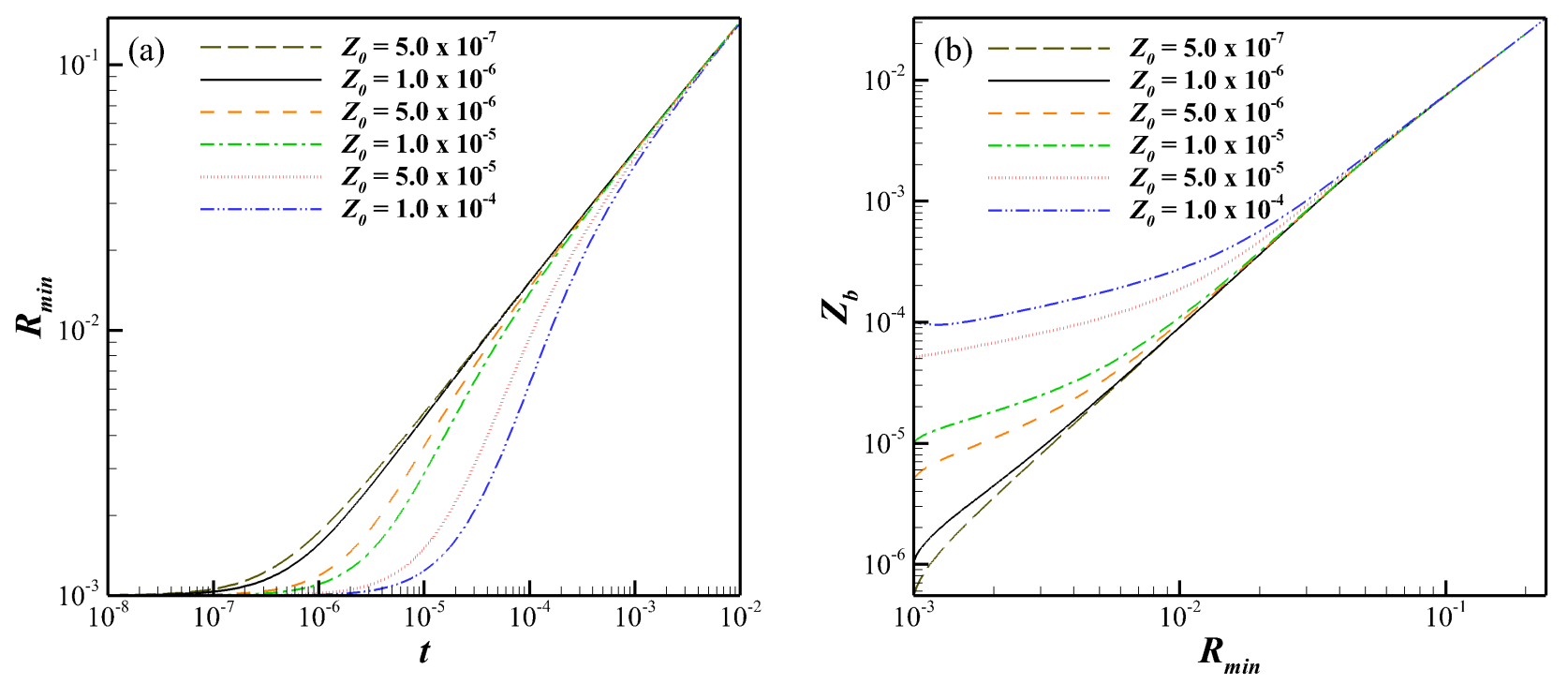

FIG. 2. The effect of the initial bridge half height $Z_{0}$ on the scaling behavior of the instantaneous values of the minimum radius $R_{\text {min }}$ and the half height, or the axial scale, $Z_{b}$ of the neck: the variation of (a) $R_{\text {min }}$ with time $t$ and (b) $Z_{b}$ with $R_{m i n}$. In all simulations, the initial bridge radius $R_{0}=10^{-3}$ and $O h=0.1$.

values of $Z_{0}$, the simulations appear to indicate the possibility of two scaling regimes and a transition between them, which is a point that is returned to in the paragraph after the next one. However, the initial regime disappears as the value of $Z_{0}$ is systematically decreased, thereby revealing that it is a computational artifact owing to using too large a value of $Z_{0}$. It is worth noting that the scaling results for the two smallest values of $Z_{0}$ shown in Fig. 2(a) virtually lie on top of one another during the entire duration of coalescence. Therefore, for simulation results to be insensitive to the value of the initial bridge height, $Z_{0} \approx R_{0}^{2}$. Moreover, when the simulation results are independent of the size of the initial bridge, it is found that once initial transients have decayed, $R_{\min } \sim t^{1 / 2}$, in accord with experiments [25] and theory $[26]$.

Figure 2(b) shows the variation of the axial scale $Z_{b}$ with the minimum neck radius $R_{\text {min }}$ for the same set of simulations as in Fig. 2(a) where the initial bridge radius $R_{0}$ is held fixed at $10^{-3}$ but the initial bridge half height $Z_{0}$ is varied. This figure makes plain that the curves depicting the variation of $Z_{b}$ with $R_{\min }$ for different values of $Z_{0}$ all fall on top of one another once sufficient time has elapsed and that the overlap between the various simulations starts earlier and earlier as the bridge half height $Z_{0}$ decreases. Once again, the scaling results for the two smallest values of $Z_{0}$ shown in Fig. 2(b) virtually lie on top of one another during the entire duration of coalescence. Once the initial transients have decayed, the results depicted in Fig. 2(b) show that $Z_{b} \sim R_{\text {min }}^{2}$, a finding that also accords with experiments $[25]$ and theory [26] and is a consequence of the idealized picture of coalescence where a neck of radius $R_{\min }$ and height $R_{m i n}^{2}$ grows on two touching spheres of unit radius (see Hopper [5, 6], Eggers et al. [7], Duchemin et al. [9], and Paulsen et al. [14]). Figure 2(a) and (b) show that the duration of the initial transients can be minimized by using an initial bridge of sufficiently small height and that the onset of the attainment of the physically correct scaling that the axial scale varies as the square of the radial scale can occur virtually from the beginning of the simulations by selecting the initial value of the bridge half height $Z_{0}$ to be given by $Z_{0} \approx R_{0}^{2}$. Henceforward, all simulation results to be reported are obtained using $Z_{0}=R_{0}^{2}$.

We now return to the occurrence of the two scaling regimes in figure 2(a) the first of which has already been shown to be a computational artifact due to the use of a too large of a value of the initial bridge height. Indeed, the two scaling regimes and the transition between them can be understood by realizing that the first is an initial Taylor-Culick [36-38] regime with $r \sim t$ on a sheet of constant thickness $Z_{0}$ that gives way to the second regime with $r \sim t^{1 / 2}$ on a sheet of thickness $z \sim r^{2}$.

Next, the effect of the initial value of the bridge radius $R_{0}$ on the computed variation of $R_{\min }$ with $t$ is examined. Although Fig. 3 shows that systematic halving of the value of $R_{0}$ pushes the period of the existence of initial transients to earlier times and decreases their duration, all three simulations can be seen to lie on top of one another and that $R_{\min } \sim t^{1 / 2}$ once all the transients have died down. Therefore, all simulation results that are hereafter reported have been obtained using $R_{0}=10^{-3}$ unless otherwise indicated. 


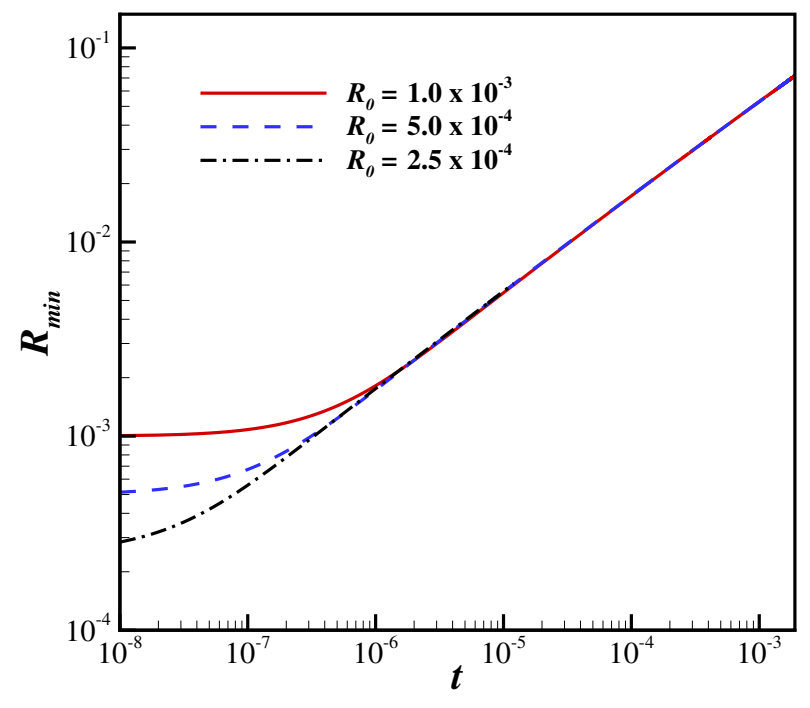

FIG. 3. The effect of the initial bridge radius $R_{0}$ on the scaling behavior of the minimum neck radius. In all three cases, the initial bridge half height is related to the initial bridge radius as $Z_{0}=R_{0}^{2}$ and $O h=3 \times 10^{-3}$.

\section{RESULTS AND DISCUSSION}

\section{A. Interface Shapes, Flow Fields, and Radial and Axial Scalings}

Simulations readily enable visualization of a number of features of the dynamics such as flow fields within the liquid exterior to the coalescing bubbles, and in particular within the retracting sheet (film), that would be challenging to observe in the laboratory and, therefore, have not been examined experimentally [25]. Similarly, while the theoretical analysis carried out by Munro et al. [26] provides a wealth of information about flow fields, simulations can also reveal when and how the actual flow fields can deviate from those based on the slenderness of the retracting sheet when that assumption no longer holds at large times. Figure 4 shows the interface shapes and the instantaneous streamlines and pressure contours near the tips of receding films of two different Ohnesorge numbers: in one case (on the left), the coalescence is taking place in a highly viscous liquid of $O h=8$ while in the other (on the right), the coalescence is occurring in a slightly viscous or nearly inviscid liquid of $O h=3.97 \times 10^{-3}$. Stark differences between the two cases become immediately apparent even at early times. In the highly viscous case, the sheet recedes while retaining a profile that remains similar from one instant to the next and the pressure within it decays monotonically in the radial direction measured away from its tip. In the nearly inviscid case, however, a bulge rapidly develops at the edge of the retracting sheet and the flow field undergoes a change from uniform decay away from the tip to the development of recirculations in the radial direction along the film corresponding to waves on the interface. Interestingly, the dynamics in the nearly inviscid case is qualitatively similar to that observed during the contraction, or recoil, of nearly inviscid filaments where similar capillary waves arise (see Schulkes [39] and Notz and Basaran [30]). However, whereas the out of plane curvature eventually drives the filaments to undergo pinch-off by the end-pinching mechanism in retracting filaments, the absence of the out of plane curvature precludes the possibility of film rupture by the same mechanism in the present case.

Next, scaling laws that govern the variation with time of the radial and axial scales, viz. the minimum radius $R_{\min }$ and half height $Z_{b}$ of the growing bridge connecting the two bubbles, are determined from simulations carried out at three different values of the Ohnesorge number, as shown in Fig. 5(a) and (b). First, regardless of whether the liquid is nearly inviscid, moderately viscous, or highly viscous, Fig. 5(a) shows that once initial transients have decayed, $R_{\min } \sim t^{1 / 2}$ in all three cases. While the radial scaling exponent is the same in each case, the prefactor relating $R_{\min }$ and $t$ are different. This is a point that is returned to below. Similarly, Fig. 5 (b) shows that once initial transients have died down, $Z_{b} \sim R_{\min }^{2}$ in all three cases. As an independent way of determining the axial scaling that differs from that presented in the previous section, the variation with time of the planar curvature evaluated at $z=0, H_{i n}=\frac{\partial^{2} r}{\partial z^{2}}$, is also evaluated from the simulations. For all three values of the Ohnesorge number, Fig. 5(c) shows that the computed value of $H_{\text {in }} \sim R_{\text {min }}^{-3}$ or that $H_{\text {in }} \sim t^{-3 / 2}$. This prediction accords with the scaling results given in Fig. 5(a) and (b) because based on those results, the inplane curvature evaluated at $z=0$ is expected to scale as $H_{\text {in }} \sim r / z^{2} \sim R_{\text {min }} /\left(R_{\text {min }}^{2}\right)^{2} \sim R_{\text {min }}^{-3}$.

The experimental measurements of Paulsen et al. [25], the asymptotic analysis of Munro et al. [26], and the simulation results of this paper all show that the evolution of the minimum neck radius follows a power-law relationship of the form $R_{\text {min }}=B t^{1 / 2}$ where $B$ is a prefactor. Moreover, all three techniques reveal the existence of two distinct dynamical regimes, one that can be categorized as an inertial regime which arises when $O h \ll 1$ and the other a viscous regime which occurs when $O h \gg 1$. While the power-law exponents in both regimes are the same, the prefactors are quite different as made evident by Fig. 5 (a). In previous studies, it has proven convenient to determine and present these prefactors by using two different non-dimensionalizations each of which is more appropriate in one or the other of the two regimes. Thus, for low Ohnesorge number fluids, it proves convenient to make time dimensionless using the inertial-capillary time, $t_{I}=\sqrt{\rho \tilde{R}^{3} / \gamma}$, as in section II of this paper, but for high Ohnesorge number fluids, it is advantageous to use the visco-capillary time, $t_{V}=\mu \tilde{R} / \gamma$, as the characteristic time scale in the non-dimensionalization. This 

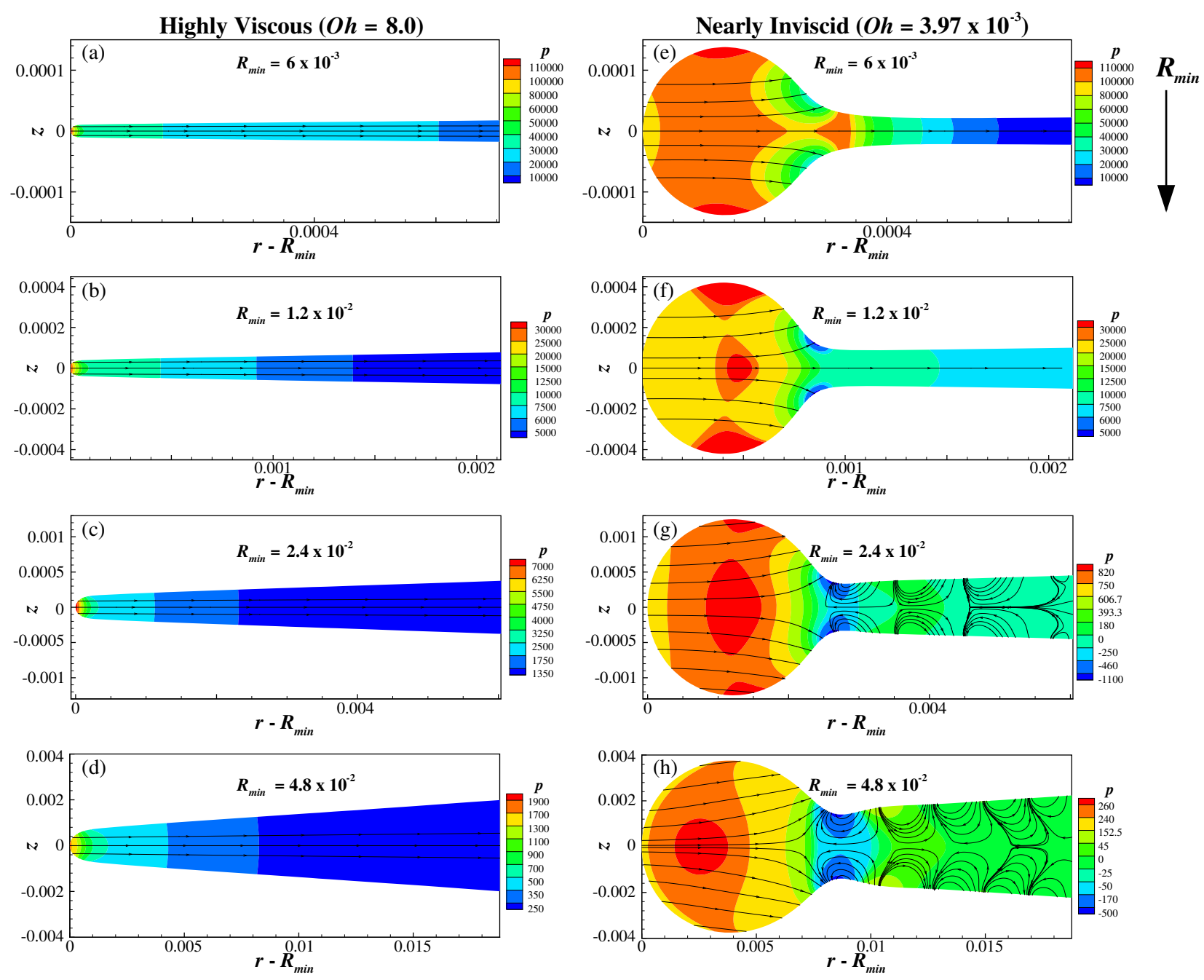

FIG. 4. Transient profiles of retracting sheets and instantaneous streamlines and pressure contours within them. In the figure, the radial coordinate has been shifted by the instantaneous value of the minimum radius so that all profiles begin at zero. Here, interface profiles and flow fields in the left column, (a)-(d), correspond to a highly viscous sheet and the ones in the right column, (e)-(h), correspond to a nearly inviscid sheet. The value of the minimum neck radius for each snap shot in time is shown on the figure. The values of the pressure contours for each instant in time are shown next to the corresponding figure. The $R_{\text {min }}$ and the down arrow at the top right indicate the direction of increasing $R_{m i n}$ and hence time. In both simulations, the initial bridge radius and half height are given by $R_{0}=10^{-3}$ and $Z_{b}=R_{m i n}^{2}$.

approach is also adopted here and results in the following two forms of the scaling law governing the variation of the dimensionless minimum neck radius with dimensionless time:

$$
\begin{aligned}
& R_{\text {min }}=B_{I}\left(\frac{\tilde{t}}{t_{I}}\right)^{1 / 2} \\
& R_{\text {min }}=B_{V}\left(\frac{\tilde{t}}{t_{V}}\right)^{1 / 2}
\end{aligned}
$$

Here, Eq. (3a) is the form of the scaling relation that is appropriate in the inertial regime, with $B_{I} \equiv B_{I}(O h)$ the prefactor that results when $t_{I}$ is used to make time dimensionless. Equation (3b), on the other hand, is the form of the scaling relation that is appropriate in the viscous regime, with $B_{V} \equiv B_{V}(O h)$ the prefactor that results when $t_{V}$ is used to make time dimensionless. The two prefactors $B_{I}$ and $B_{V}$, which are hereafter referred to as the inviscid and the viscous prefactors, are simply related as $B_{V}=O h^{1 / 2} B_{I}$. Most interesting are the limiting values of the prefactors as $O h$ approaches zero or infinity, which are denoted by $B_{I}(0)$ and $B_{V}(\infty)$. Fits to data from experimental measurements [25] give values of 1.4 and 1.2 for $B_{I}(0)$ and $B_{V}(\infty)$ while asymptotic analysis [26] predicts values of 1.81 and 0.89 for the limiting values of the two prefactors.

Simulations were carried out for a range of values of $O h$ and the results on the variation of the minimum ra- 

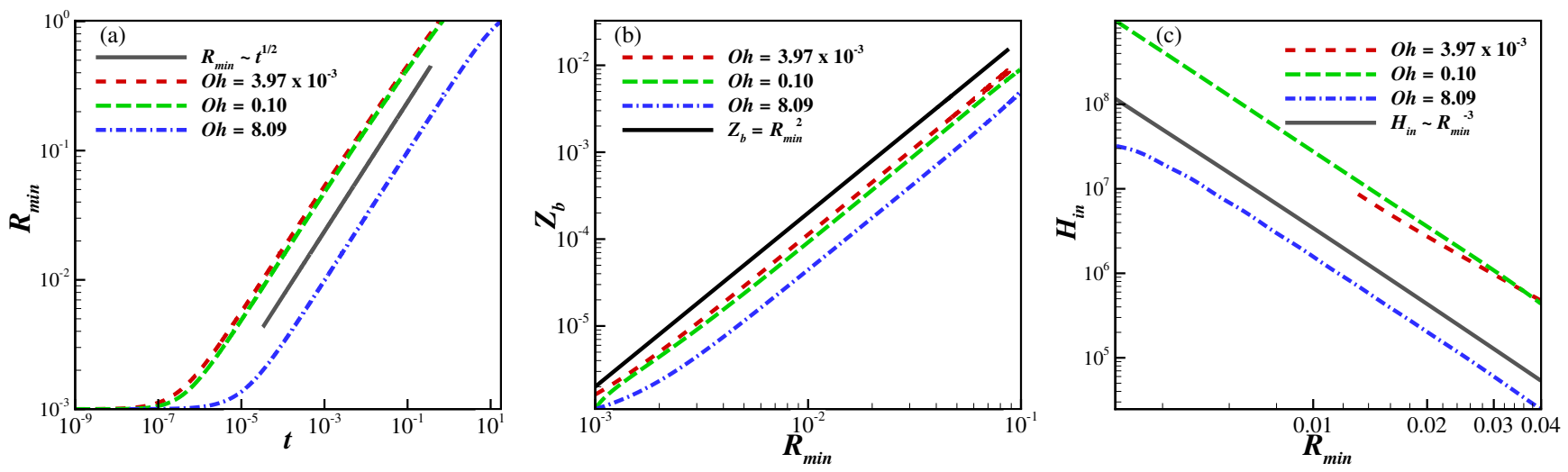

FIG. 5. Scaling behavior of three quantities obtained from simulations: the variation of (a) the minimum neck radius $R_{m i n}$ with time $t$, (b) the neck/bridge half height or the axial scale $Z_{b}$ with $R_{m i n}$, and (c) the in-plane curvature $H_{i n}$ with $R_{m i n}$. In all three parts, simulation results are shown for three different values of $O h$. In parts (a)-(c), lines of slopes of $1 / 2,2$, and -3 are also shown to demonstrate the scaling that is followed by the results once the initial transients have died out. In all simulations, initial conditions are such that $R_{0}=10^{-3}$ and $Z_{0}=R_{0}^{2}$.

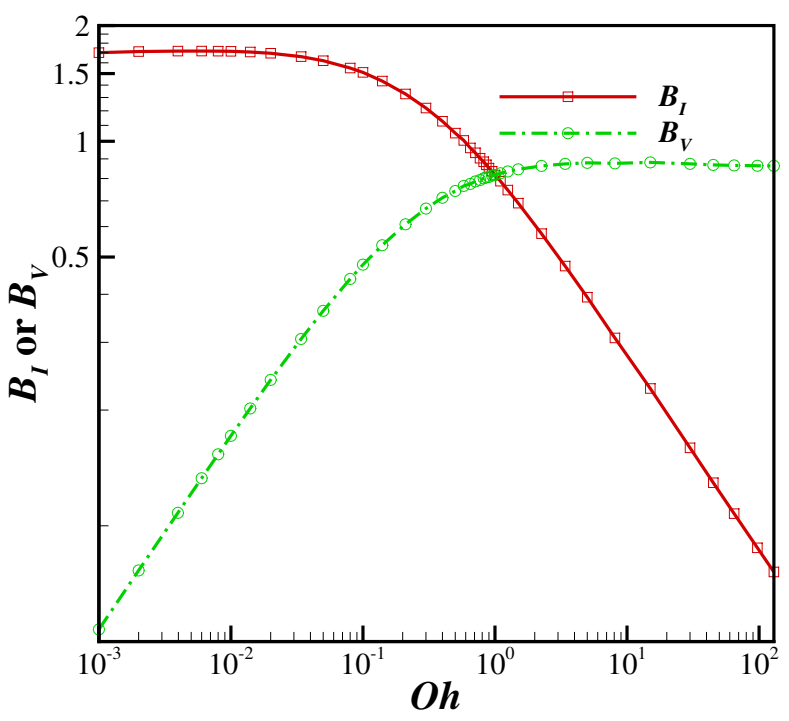

FIG. 6. Variation with $O h$ of the inviscid prefactor $B_{I}$ and the viscous prefactor $B_{V}$ obtained from simulations. When plotted together, the results make plain the transition that occurs from the inertial to the viscous regime as Ohnesorge number is varied from small to large values and as the appropriate characteristic time scale switches from the inertial-capillary time to the visco-capillary time.

dius $R_{\min }$ with time $t$ were fitted to extract the values of the prefactors from simulation data. The resulting prefactors as a function of $O h$ are presented in Fig. 6 and clearly show that there are indeed two regimes where either $B_{I}$ or $B_{V}$ remains constant. The results of Fig. 6 also reveal that the value of the Ohnesorge number where the crossover between the two regimes occurs is around $O h \approx 1$, a value that is in good agreement with the value of $O h \approx 0.7$ obtained by Paulsen et al.[25] in their experiments. The simulations also show that $B_{I}$ approaches 1.71 as $O h \rightarrow 0$ and that $B_{V}$ approaches 0.87 as $O h \rightarrow \infty$.

Clearly, the limiting values of the prefactors obtained from the simulations are in much closer agreement with the theoretical values obtained by Munro et al. [26] than the experimental values obtained by Paulsen et al. [25]. The very good agreement between computational and theoretical predictions, and the substantial deviation of the computationally and theoretically obtained prefactors from the experimentally measured ones indicate that the discrepancies are most likely due to the inability of the experiments to be able to probe the dynamics at early times when the neck radii are too small to be measured with the optical method available in the laboratory. This hypothesis can easily be tested by limiting the range of values of the minimum neck radius from simulations to be fitted to that which is accessible in experiments, viz. limiting the range of $R_{\min }$ to $0.2 \leq R_{\min } \leq 0.6$. By so limiting the fits to simulation results, it is found that in the limit as $O h \rightarrow 0$, the computed value of $B_{I}$ approaches 1.45 whereas in the limit as $\mathrm{Oh} \rightarrow \infty$, the computed value of $B_{V}$ approaches 0.85 . Thus, in the inviscid limit $(O h \rightarrow 0)$, simulations accord well with theory for sufficiently small values of $R_{\min }$ and when the dynamics is in the vicinity of the space-time coalescence singularity whereas at later times, the dynamics begins to depart from the scaling behavior predicted from theory and the scaling that is predicted from simulations falls in line with what is observed in experiments. By contrast, in the viscous limit $(O h \rightarrow \infty)$, the prefactor obtained from simulations for $R_{\min } \ll 1$ and the prefactor that is obtained from simulations using larger values of $R_{\min }$ are virtually identical and agree with the prefactor obtained from 
theory. The deviation of $B_{I}(0)$ obtained from simulations for larger values values of $R_{\text {min }}$ from its asymptotic value as $R_{\min } \rightarrow 0$ is most likely due to the loss of slenderness in the receding film, an expectation the validity of which will be tested in later sections. However, the aforementioned reason cannot explain why the limiting value of the viscous prefactor as $\mathrm{Oh} \rightarrow \infty$ obtained from simulations even using larger values of $R_{\text {min }}$ differs substantially from the value obtained from experiments. Part of the discrepancy between the value predicted from simulations (0.85) and that measured in the experiments (1.2) may be attributable in this case to the larger uncertainty involved in the calculation of the relevant timescales of coalescence for large $O h$ cases presented in Paulsen et al. [25] which are subsequently used in determining the values of the prefactors in the scaling relations.

While the agreement between simulations and theory is quite good, the discrepancy of $6 \%$ in the limiting value of the inviscid prefactor $B_{I}(0)$ between them is non-trivial. Two possible reasons for this discrepancy are either that the simulations, despite accessing earlier times than experiments, are also unable to probe the very early times in the aftermath of the singularity or that some of the assumptions of the theoretical analysis become less valid more quickly than expected as time increases. To gain insight into the aforementioned discrepancy between simulation and theory, a new computational approach is presented in the next subsection in which the liquid domain $\Omega(t)$ is truncated so that most of the computational power can be focused on the important thin film region between two coalescing bubbles. The truncated domain approach to be presented will allow probing of the dynamics by simulation for neck radii much smaller than what has been possible in the computational results presented up to this point in the paper.

\section{B. Truncated Domain}

The difficulty in simulating bubble coalescence is caused by the multi-scale nature of the problem in that in a given simulation one must resolve phenomena occurring over length scales ranging from the smallest scale, which is of the order of the bridge height or $O\left(R_{0}^{2}\right)$, to the largest scale, which is of the order of the bubble radii or $O(1)$. A way to circumvent this difficulty can be gleaned from an examination of the variation with time, or equivalently with $R_{\min }(t)$, of the radial distance $\delta r$ that is required for the radial velocity evaluated at $z=0$ in the retracting sheet to fall to $10 \%$ of the value $U_{\max }$ it has at the sheet's tip, viz $\delta r=r\left(u=0.1 U_{\max }\right)$. As shown in Fig. 7 , this distance is at most of $O\left(R_{\min }\right)$ and remains proportional to $R_{\min }$ until the neck reaches macroscopic dimensions. Thus, it should be possible to model accurately the growth of the bridge connecting the two bubbles without having to simulate the flow in the entire liquid region $\Omega(t)$ exterior to the bubbles. Therefore, the domain exterior to the neck is truncated at some radial

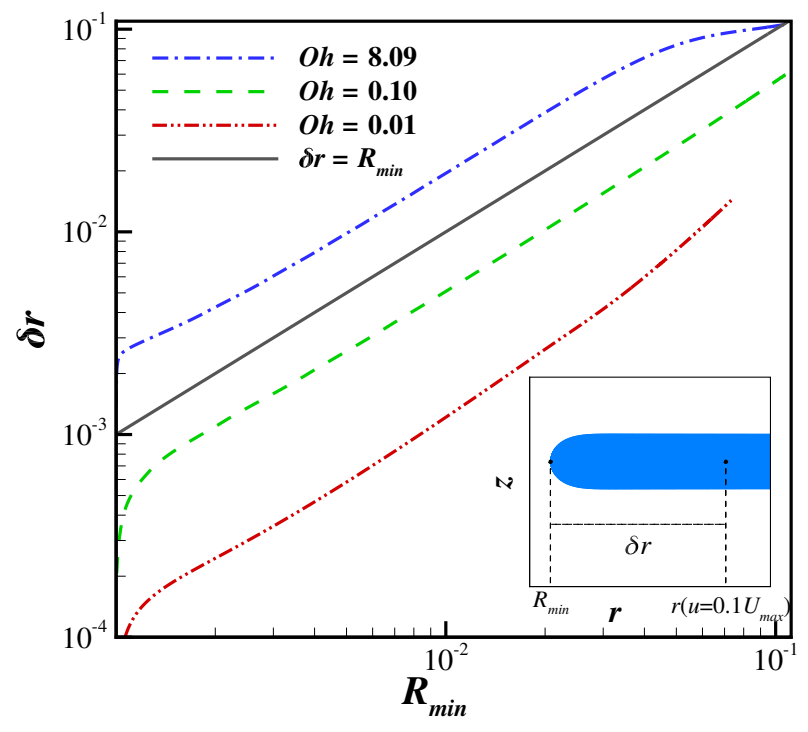

FIG. 7. Variation with $R_{\text {min }}$ of the dominant length scale $\delta r$ in the radial direction. $\delta r$ is the length measured from the receding tip for which the radial velocity in the sheet falls to $10 \%$ of its value at the tip. Regardless of the value of $\mathrm{Oh}, \delta r$ is linearly proportional to $R_{\min }$.

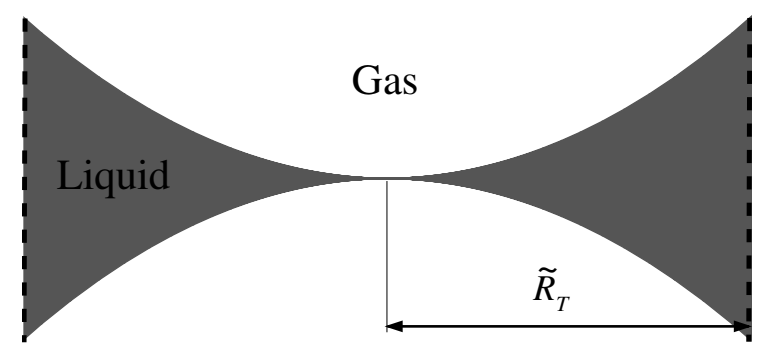

FIG. 8. Truncated domain: definition sketch. The thin sheet between the coalescing bubbles is cut off at a radial distance $\tilde{R}_{T}$ and the region exterior to this domain is excluded from the simulations.

distance $r=R_{T}=\tilde{R}_{T} / \tilde{R}$. The value of $R_{T}$ is picked such that the end of the domain is sufficiently far from the tip of the receding film so that its location will not affect the retraction dynamics but not so large that the end of the domain is well within the film region in the two bubble coalescence problem, viz. $R_{0} \ll R_{T} \ll 1$. Figure 8 shows the new truncated domain where the only new parameter that is introduced into the analysis is the truncation radius $R_{T}$ which merely gives the radial location where the film is truncated. The vastly reduced size of the new truncated domain compared to the original domain $\Omega(t)$ makes it possible to start the simulations from much smaller values of the initial neck radius $R_{\min }(0)=R_{0}$ and also to concentrate the elements where they are needed most in the computations.

With the truncated domain, boundary conditions on 
the flow field are required at $r=R_{T}$. Two options were considered. In the first one, a stress free boundary condition was imposed at $r=R_{T}$. In the second one, a zero velocity boundary condition was imposed at $r=R_{T}$. No discernible differences in the simulation results were observed when computational data obtained with the two different boundary conditions were compared against one another. Therefore, the zero velocity boundary condition was used in all cases because of the ease of implementation. Since even in the purely viscous limit where the relevant radial length scale $\delta r$ in the film is largest, $\delta r$ is still only of the order of $R_{\min }$. Thus, a truncation radius equal to $100 R_{\min }(0)=100 R_{0}$ has been found through computational experiments to be sufficiently large as to not impact the dynamics of the receding film. In computations involving the truncated domain, simulations were stopped before the motion of the receding film begins to be influenced by the location of the artificial boundary located at $r=R_{T}$. Therefore, while the truncated domain approach enables probing the dynamics at very early times, it does not allow following the dynamics into late times when the two bubbles have nearly merged.

In order to verify the accuracy of the proposed truncated domain approach, computational predictions made with it were compared against ones using the entire liquid domain. The results of such comparisons will be highlighted with a specific example that involves bubble coalescence at the relatively small value of the Ohnesorge number of $O h=3 \times 10^{-3}$ as our primary interest in using the new truncated domain approach is to better investigate the inviscid limit. In this test, $R_{0}=10^{-3}$ and $Z_{0}=R_{0}^{2}$. A comparison of the scaling results obtained with the two approaches is presented in Fig. 9 and shows excellent agreement between them, thereby providing confidence for the use of truncated domain approach for carrying out parametric studies. In the simulation results to be reported hereafter, a domain length of $R_{T}=100 R_{0}$ was used to give at least a full decade of scaling data for fitting once the initial transients have died out and before the film has receded far enough such that the artificially located boundary begins to affect the dynamics.

To demonstrate the power of the truncated domain approach, sets of simulations were carried out by fixing $O h$ and starting each simulation at a successively lower value of the minimum neck radius. Figure 10 shows the typical outcome albeit for the situation when $O h=3 \times 10^{-3}$. Thus, a simulation was started with $R_{0}=10^{-3}$ and continued until $R_{\min }$ increased by more than an order of magnitude but less than two orders. The process was repeated by starting simulations with a value of $R_{0}$ one tenth this value, followed by another simulation where the value was one hundredth of this value, and so on until terminating with a simulation where the initial value of the minimum neck radius was $10^{-7}$. As shown in Fig. 10 , despite the fact the simulations were started from different values of $R_{0}$, once initial transients decayed, the data overlapped and fell on top of a line of slope $1 / 2$ on a

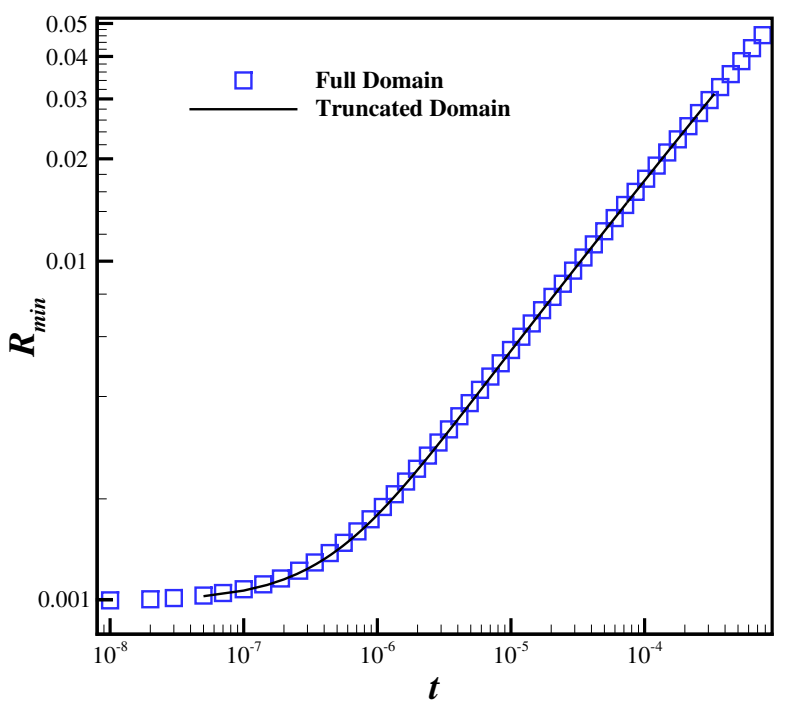

FIG. 9. Variation of $R_{\text {min }}$ with $t$ : comparison of results on radial scaling obtained from simulations using the entire domain $\Omega(t)$ (open square symbols) and the truncated domain (solid line). Here, $R_{0}=10^{-3}, Z_{0}=R_{0}^{2}$, and $O h=3 \times 10^{-3}$.

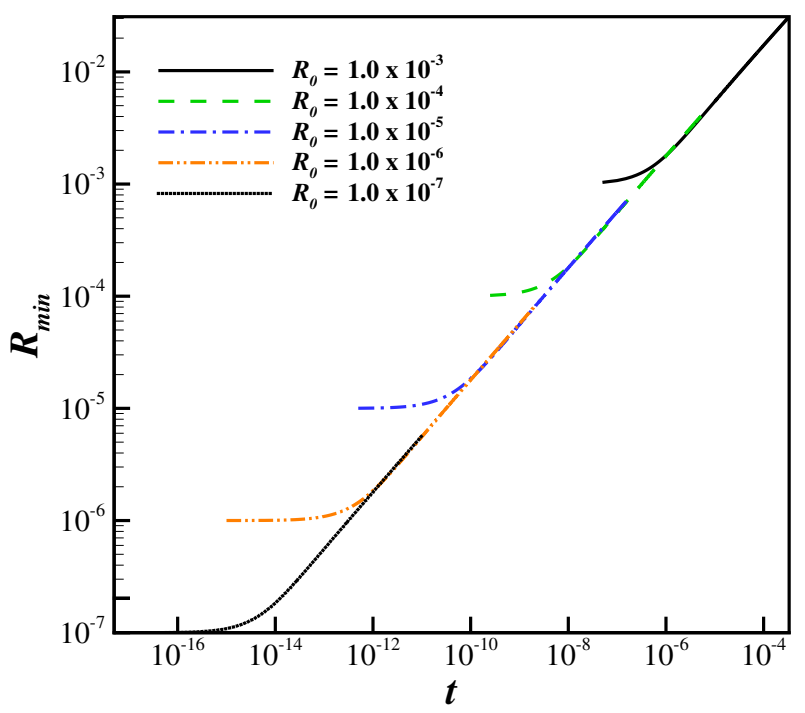

FIG. 10. Variation of $R_{\min }$ with $t$ obtained from different simulations using the truncated domain approach. The different simulations start from initial conditions using different values of the initial bridge radius $R_{0}$ but with $Z_{0}=R_{0}^{2}$. After the decay of initial transients, each simulation falls on a line of slope $1 / 2$. As explained in the text, the different simulations can then be stitched together to allow simulating drop coalescence from extremely early times when the neck radii are orders of magnitude smaller than the bubble radii. In all simulations $O h=3 \times 10^{-3}$. 


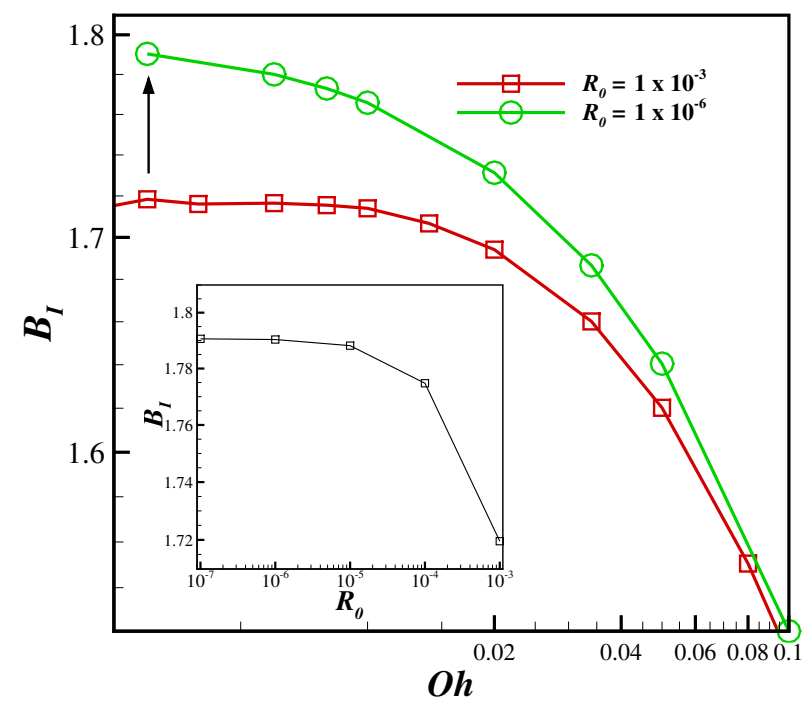

FIG. 11. Variation of the inviscid prefactor $B_{I}$ with the Ohnesorge number $O h$ for simulations carried out with two different initial conditions of $R_{0}=10^{-3}$ and $R_{0}=10^{-6}$ but where $Z_{0}=R_{0}^{2}$ in both cases. The arrow indicates how $B_{I}$ would change if the value of $R_{0}$ is lowered by three orders of magnitude. This change is detailed in the inset which shows the variation of $B_{I}$ with $R_{0}$ for $Z_{0}=R_{0}^{2}$ when the Ohnesorge number is held constant at $O h=3 \times 10^{-3}$.

$\log -\log$ plot of $R_{\min }$ versus $t$. With the truncated domain approach and stitching together data from different simulations as shown in Fig. 10, one could go to arbitrarily smaller and smaller values of the initial neck radius and produce $R_{\min }$ versus $t$ data spanning as many orders of magnitude as desired.

Next, the effect of lowering $R_{0}$ from $10^{-3}$ to $10^{-7}$ on the inviscid prefactor in the zero-Oh or inviscid limit was investigated. The results of such simulations are shown in the inset to Fig. 11 for $O h=3 \times 10^{-3}$. At this value of $O h$, lowering the value of $R_{0}$ below $10^{-5}$ produced no discernible changes in the value of $B_{I}$. With these calculations, the value of $B_{I}$ determined from simulations in the zero Ohnesorge number limit increased to 1.79 , which is within $1 \%$ of the value predicted from Munro et al.'s theory [26]. With the initial bridge size no longer an issue, next the value of $O h$ was systematically reduced, as shown in the main part of Fig. 11. Although this figure does show the inviscid prefactor in the zero-Oh limit being approached by the simulation data for sufficiently small $\mathrm{Oh}$, with one additional simulation carried out for values of $O h$ and $R_{0}$ both smaller than those shown in this figure, viz. when $O h=5 \times 10^{-4}$ and $R_{0}=10^{-7}$, the computed value of the inviscid prefactor in the zero- $O h$ limit increased ever so slightly to 1.80 . This value, which we take to be the value of $B_{I}(0)$ predicted from simulations, is within about $0.5 \%$ of the value predicted from theory.

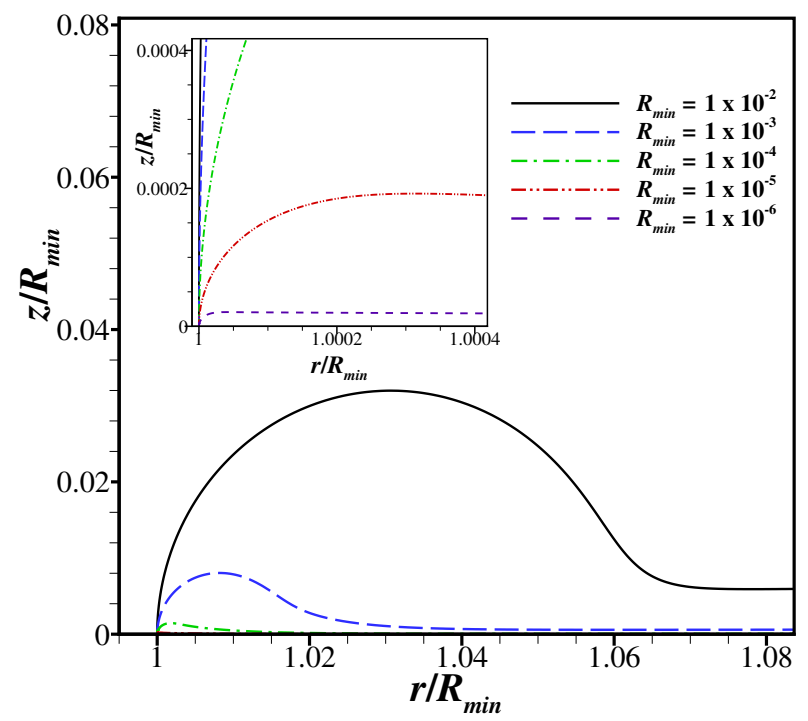

FIG. 12. Computed evolution in time of the profiles of the receding film when $O h=3 \times 10^{-3}$. For each profile shown, both the axial and the radial coordinates have been normalized by the minimum radius at that instant in time. The inset shows zoomed-in views of the tips of the film and makes plain that the profiles at the two earliest times, i.e. for the smallest two values of $R_{\text {min }}$, are still slender. Both the main figure and the inset highlight the transition that occurs at later times from profiles that are slender to ones where the retracting tips have become bulged. All shapes have been obtained from different simulations where $R_{0}$ is one order of magnitude smaller than the value of $R_{\min }$ for which a given profile is shown. Also, $Z_{0}=R_{0}^{2}$ in each simulation.

With the truncated domain approach and using a set of simulations each of which is started with a different value of $R_{0}$, results can be stitched together to investigate why a shift from the theory is seen in the simulations at later times by first looking at the overall evolution of the film profile near the tip of the receding film. The results of such simulations are shown in Fig. 12 when $O h=3 \times 10^{-3}$. These results show clearly that there is a transition from initial profiles that are slender to ones which exhibit rather large bulges. This loss of slenderness is likely the culprit behind the deviations from theory: indeed, the loss of slenderness for $R_{\text {min }} \approx 10^{-4}$ seen in Fig. 12 coincides with the departure of the prefactor from its limiting value obtained in the limit of $R_{0} \rightarrow 0$ seen in the inset to Fig. 11.

It is noteworthy, however, that the afore-mentioned departure of the prefactor at finite $R_{\min }$ from its asymptotic value in the vicinity of the singularity is relatively modest despite the loss of slenderness in the receding film. Indeed, it was surmised by Munro et al. that the fluid contained within the entire growing bulge virtually moves as a plug at the same velocity as the retracting tip. This hypothesis can now be rigorously tested by 
evaluating the variation of the radial velocity in the volume contained within the bulge near the tip. Here, this volume $V_{b}$ is taken to be the region of fluid from the tip to the radial position at which the bulge is widest. The variation in the radial velocity in the bulge is then calculated as $U_{v}=\left(1 / V_{b}\right) \int_{V_{b}}\left|U-U_{\text {com }}\right| d V$ where $U_{\text {com }}$ is the velocity of the center of mass of the fluid within $V_{b}$. This variation in the radial velocity with time as $R_{\text {min }}$ increased between one and two orders of magnitude was evaluated for the two cases of $R_{0}$ of $10^{-3}$ and $10^{-6}$ with an $O h$ of $3 \times 10^{-3}$. As expected, for the case with the lower value of $R_{0}$ and where the interface profile is slender, the average deviation from $U_{\text {com }}$ is of the order $0.01 \%$ which, for all practical purposes, is negligible. For the case with the higher value of the initial neck radius, the deviation was substantially larger than that in the first case but still remained below $2 \%$. A fairly large number of simulations revealed that the assumption of a blob moving essentially at a constant velocity was fairly reasonable even for neck radii as large as $R_{\text {min }} \approx 10^{-2}$.

Munro et al. [26] determined the similarity profiles of receding sheets directly in their analysis. Here, the selfsimilar profiles will be determined by suitably collapsing the transient profiles obtained from the solution of the Navier-Stokes and continuity equations. Therefore, the collapse of the transient profiles will be accomplished by rescaling the radial and axial length scales by $R_{\min }$ and $R_{\text {min }}^{2}$, respectively. First, to emphasize the main differences between coalescence at small and large Ohnesorge numbers, we plot in Fig. 13 the collapsed interface profiles for a range of Ohnesorge numbers at the same value of $R_{\text {min }}=3 \times 10^{-2}$. This figure shows that for large $O h$ the scaled solutions for different values of $O h$ appear to collapse onto a single profile, a point that is returned to below. The scaled profiles for large $O h$ and in particular in the limit as $O h \rightarrow \infty$ are slender as expected. The scaled profiles at small $O h$, however, are quite different from their counterparts at large $O h$ and all exhibit a bulge at the leading edge of the sheet that is the trademark of the dynamics of sheet retraction for low-viscosity fluids.

To demonstrate the collapse of transient solutions onto a similarity profile, we plot transient solutions as $t$ or $R_{\text {min }} \rightarrow 0$ at a single value of $O h$ after rescaling the radial and axial coordinates as just described. Figure 14 shows a number of scaled profiles when $O h=0.9$ and clearly demonstrates the collapse of the rescaled transient profiles onto a single similarity profile as $R_{\min } \rightarrow 0$. It is noteworthy that the scaled profiles for the two smallest values of $R_{\min }$ virtually fall on top of another and are indistinguishable in the figure.

As with the interface shape, the radial velocity, when suitably rescaled, is also expected to exhibit selfsimilarity as $t$ or $R_{\text {min }} \rightarrow 0$. Here, we rescale the radial velocity along the plane of symmetry $z=0$ by its maximum value $U_{\max }$, which is always found to occur at the location where the neck radius is a minimum. Figure 15 shows the collapsed radial velocity profiles as a function

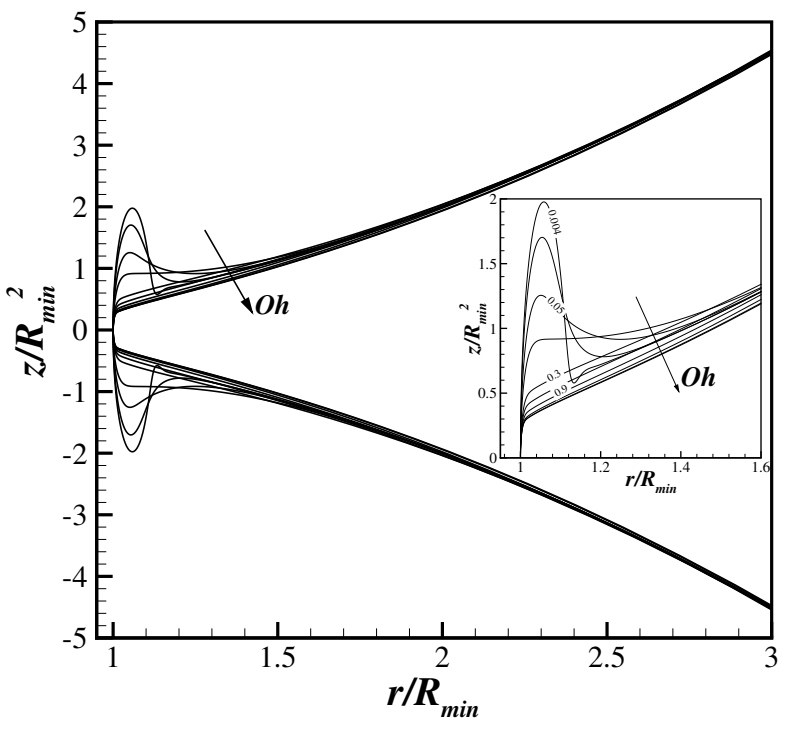

FIG. 13. Variation of appropriately scaled profiles of receding films at the instant in time when $R_{\text {min }}=0.03$ for $O h=0.004$, $0.02,0.05,0.1,0.3,0.5,0.9,8.09,15.0$, and 45.0 showing the interface profiles in the main part of the figure and zoomed-in views of the tips of the profiles in the inset. For small values of $O h$, the profiles are shown at this particular value of $R_{\text {min }}$ so as to highlight the formation of bulged tips. For moderate to large values of $\mathrm{Oh}$, the profiles depicted in both the main figure and the inset show that the scaled profiles are slender. Also, for these moderate to large values of $O h$, the scaled or collapsed profiles are virtually identical to scaled profiles obtained at earlier times or, equivalently, for smaller values of $R_{\text {min }}$. Thus, these collapsed profiles represent the similarity profiles for these values of $O h$. Also, when $O h \gg 1$, the scaled profiles vary only slightly with $\mathrm{Oh}$ and in fact collapse onto a single universal profile as $\mathrm{Oh} \rightarrow \infty$. In all simulations, $R_{0}=10^{-3}$ and $Z_{0}=R_{0}^{2}$.

of the scaled radial coordinate for several values of the Ohnesorge number at two different values of $R_{\text {min }}$, one before (a) and the other after (b) a bulge has formed in the small-Oh cases. Two distinct features characterize the solutions at large and small $O h$. As expected based on the discussion on scaled interface shapes, for both values of $R_{\text {min }}$, the scaled radial velocity profiles for large $O h$ tend to a single similarity profile as $O h \rightarrow \infty$. It is also worth noting that the length scale over which the velocity varies at large $O h$ is of the order of $R_{\text {min }}$, in accordance with Munro et al. [26]. However, as also pointed out by Munro et al., a boundary layer is present at small $O h$ where most of the velocity variation occurs over a length scale much smaller than $R_{\text {min }}$, as made clear by Fig. 15(a). The numerically collapsed profiles for both large and small $O h$ also show good agreement with the results of Munro et al. albeit only at early times (Fig. 15(a)). At later times once the bulge forms at small $O h$, the flow patterns become more complex as already seen 


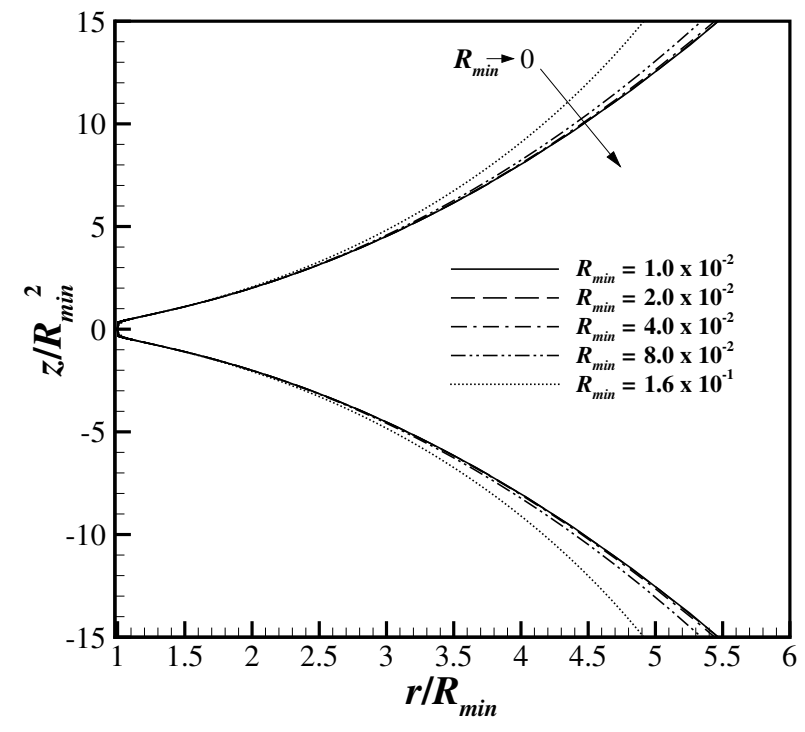

FIG. 14. Variation of appropriately scaled profiles of receding films with decreasing time. The scaled profiles are seen to collapse nicely onto a single similarity profile as $R_{\text {min }} \rightarrow 0$. Here, $O h=0.9, R_{0}=10^{-3}$, and $Z_{0}=R_{0}^{2}$.

in Fig. 4 as recirculations arise along the length of the film. Thus, Fig. 15(b) too exhibits the departure of the scaled velocity profile obtained from the simulations at the smallest value of $O h$ shown in the figure from both the corresponding similarity profile reported by Munro et al. at the same value of $O h$ and the scaled velocity profile obtained from the simulations at that $\mathrm{Oh}$ albeit at an earlier time (shown in Fig. 15(a)).

\section{CONCLUDING REMARKS}

With the detailed exploration through simulations of the parameter space governing the coalescence of two bubbles presented in this paper, the dynamics that ensues in the immediate aftermath of the instant when two bubbles have just touched has now been completely analyzed by means of experiment [25], theory [26], and simulation. All three methods have shown that the minimum radius of the neck connecting the two bubbles grows in time as $R_{\min }=B t^{1 / 2}$ where the prefactor, however, varies with the Ohnesorge number. All three studies have also revealed that two distinct regimes exist in the limit of small and large Ohnesorge numbers herein referred to as the inviscid $(O h \rightarrow 0)$ and the viscous limits $(O h \rightarrow \infty)$ each with its own characteristic value of the prefactor denoted by $B_{I}(0)$, the inviscid prefactor in the zero Ohnesorge number limit, and $B_{V}(\infty)$, the viscous prefactor in the infinite Ohnesorge number limit.

Prior to this paper, the values of $B_{I}(0)$ and $B_{V}(\infty)$ measured in the experiments and obtained from theory
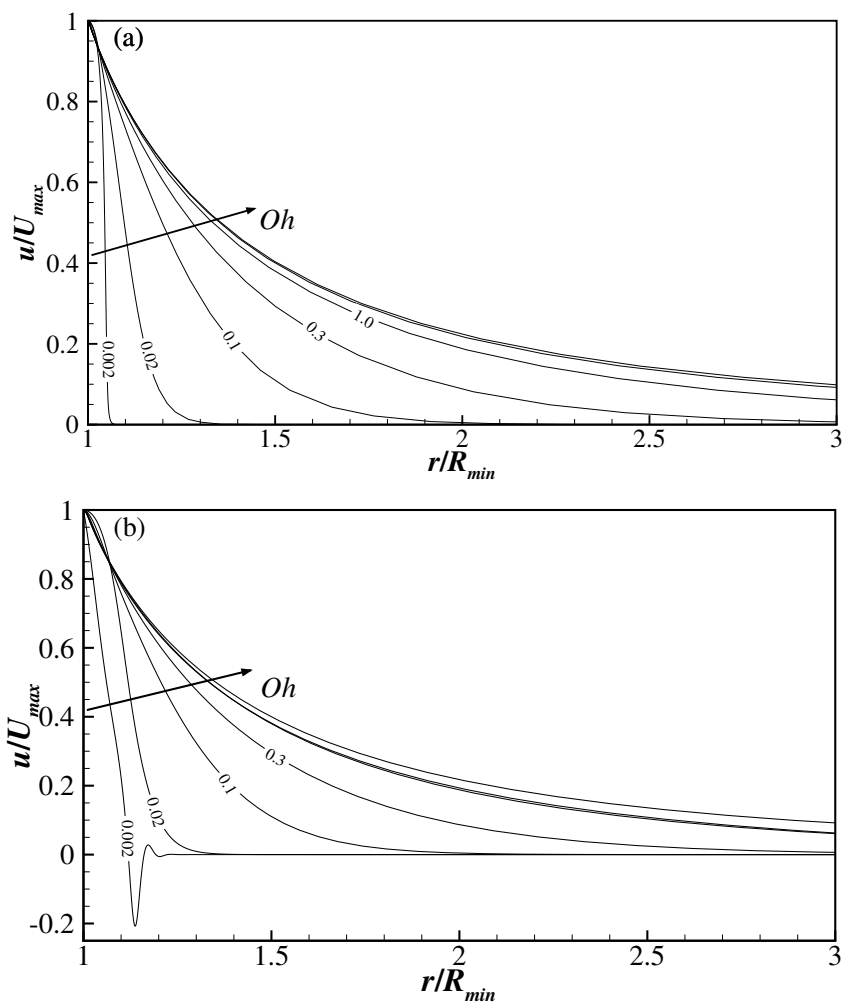

FIG. 15. Variation of the radial velocity along the plane of symmetry $z=0$ scaled by its maximum value, $u / U_{\max }$, with the scaled radial coordinate $r / R_{\min }$ : scaled velocity (a) at early times when $R_{\text {min }}=6.0 \times 10^{-3}$ and (b) later when $R_{\min }=3.0 \times 10^{-2}$. In both plots, scaled profiles are shown for $O h=0.002,0.02,0.1,0.3,1.0,3.4$, and 30.0. When $O h \ll 1$, at early times a velocity boundary layer forms (top), in agreement with the theoretical analysis of Munro et al. [26], while at later times (bottom) in the aftermath of the formation of the bulge at the tip of the retreating films, there is a distinct signature in the velocity profiles of recirculations in the film corresponding to waves on the interface. The scaled profiles when $O h \gg 1$ are virtually unchanged between the two instants shown and in both cases the profiles collapse onto a single similarity profile as $\mathrm{Oh} \rightarrow \infty$.

were not in perfect agreement with one another. Reassuringly, the limiting values of the inviscid and viscous prefactors obtained from the simulations have been found to be within $0.5 \%$ and $2.2 \%$ of the corresponding values obtained from theory. Given the excellent agreement between theory and simulations and the difficulty in accessing small neck radii in the experiments, the discrepancies in the experimentally measured values of the prefactors compared to those obtained from theory and simulation are primarily attributed to the inability of the optical technique employed in the experiments to access early times in the coalescence process when the neck radii are of the order of a micrometer or smaller. However, since the aforementioned excellent agreement between the values of $B_{I}(0)$ and $B_{V}(\infty)$ obtained from simulations and theory result from fitting simulation data for values of 
TABLE I. Values of the inviscid prefactor in the limit of zero Ohnesorge number, $B_{I}(0)$, and the viscous prefactor in the limit of infinite Ohnesorge number, $B_{V}(\infty)$, in the radial scaling laws obtained from experiment, theory, and simulation.

\begin{tabular}{lcr}
\hline \hline Study & $B_{I}(0)$ & $B_{V}(\infty)$ \\
\hline Experiment [25] & 1.4 & 1.2 \\
Theory [26] & 1.81 & 0.89 \\
Simulation $\left(R_{\min } \ll 10^{-3}\right)$ & 1.80 & 0.87 \\
Simulation $\left(0.2 \leq R_{\min } \leq 0.6\right)$ & 1.45 & 0.85 \\
\hline \hline
\end{tabular}

$R_{\text {min }} \rightarrow 0$, a fairer comparison between simulations and experiments can be carried out if the simulation data were to be fitted to the power law forms given by Eqs. (3a) and (3b) by excluding data from the simulations for values of the minimum neck radii below what is realizable in the experiments. When simulation data used in the power law fits were restricted to values of the minimum radius lying in the range $0.2 \leq R_{\text {min }} \leq 0.6$, the computed value of $B_{I}(0)$ decreased substantially from 1.80 to 1.45 , making it accord well with the experimentally measured value of 1.4. However, similarly restricting the range of values of $R_{\text {min }}$ for curve fits from simulation data at large Ohnesorge numbers resulted in a negligible change in the computed value of $B_{V}(\infty)$. The values of $B_{I}(0)$ and $B_{V}(\infty)$ obtained with the three techniques are summarized in Table I where prefactors derived from simulations include those that have been obtained by using only extremely small values of $R_{\text {min }}$ in generating curve fits (i.e. the prefactors have been obtained by only looking at $R_{\min }$ versus $t$ data for values of $R_{\min } \ll 10^{-3}$ ) and also ones that have been obtained by using values of $R_{\text {min }}$ that lie in a range that is comparable to that attainable in the experiments (i.e. the prefactors have been obtained by only looking at $R_{\text {min }}$ versus $t$ data when $R_{\text {min }}$ varies over the range $0.2 \leq R_{\text {min }} \leq 0.6$ ).

A reassuring finding reported in section III is that the initial values of the bridge radius and half height $R_{0} \ll 1$ and $Z_{0} \ll 1$ do not make much difference as to what is observed after the transients. While the duration of the initial transients can be minimized with a suitable $Z_{0}$, viz. $Z_{0} \approx R_{0}^{2}$, regardless of the values of $R_{0}$ and $Z_{0}$, it has been shown that the numerics can be relied on to give good predictions of the $t^{1 / 2}$ scaling behavior for the variation of the minimum neck radius with time. An equally important message from these results is that whatever the local mechanism, e.g. van der Waals forces, first ruptures the fluid film to start the coalescence process in a real experiment, that initial condition does not make much difference to the subsequent evolution of the bubble coalescence process.

In this paper, a new technique referred to as the truncated domain approach has also been developed. This approach has made it possible to start the simulations from an initial state when the radius of the microscopic bridge connecting the two bubbles is at least six orders of magnitude smaller than the radii of the bubbles. The truncated domain approach not only has enabled probing the very early stages of the dynamics but it has also allowed vast reductions in computation times because it does not waste valuable computational resources solving for the flow outside the thin film region which has virtually no effect on the flows occurring in the vicinity of the expanding neck. The truncated domain approach has made it possible, among other things, the accurate evaluation of the inviscid prefactor in the scaling law relating the minimum radius and time. The truncated domain approach has also enabled us to capture the transition in the low-viscosity limit from a state where the retracting film is slender to one where the tip of the film has developed a bulge. Being able to access early times has also allowed the construction of similarity solutions by appropriately collapsing transient solutions for the interface shape and fluid velocity within the retracting sheet obtained from the computations. One drawback of this technique is that an individual simulation can only capture a portion in time rather than the entire duration of the coalescence process. However, it has been shown that this drawback is easily remedied by stitching together individual simulations that are started from initial conditions corresponding to different values of the minimum bridge radius. Moreover, the method is much more versatile than this particular implementation in that if a single simulation is desired to span the period from very early to relatively late times, the size of the truncated domain can be increased dynamically in proportion to the increasing radius of the growing neck connecting the two bubbles. This approach is summarized and example simulation results obtained with it are presented in Appendix A.

Two questions immediately arise based on the results of this paper. One pertains to whether the dynamics of the fluid within real bubbles, which has been neglected here, can influence the fluid dynamics of the bubble coalescence singularity. In Appendix B, we present results of preliminary simulations that show that accounting for the flow of the gas within the bubbles has no effect whatsoever on the scaling laws of bubble coalescence. These preliminary simulations in turn lead to the second question as to how the dynamics would change if instead of gas bubbles the dispersed phase were a liquid of arbitrary density and viscosity. Paulsen et al. [25] have already studied experimentally the physics of the coalescence singularity when two viscous drops coalesce in a second viscous fluid. Thus, a goal of future research is to directly simulate the two-drop coalescence problem by using the extended version of the algorithm that has been employed to perform the simulation results reported in the appendix. Such simulations, however, are considerably more complex and costly than the ones reported here as the domains both interior and exterior to the drops have to be discretized and the governing equations have to be solved in both phases to simulate the dynamics. A report on the results of such studies will form the subject of a future communication. 
The two most important hydrodynamic singularities that arise in free surface flows of drops and bubbles are the pinch-off and the coalescence singularities involving the separation of a fluid into two pieces and the joining two pieces of fluid (see Ref. [40], page 925). Aside from being of theoretical interest, a good understanding of the nature of these singularities and having the capability to accurately simulate the dynamics in the vicinity of them would allow the accurate prediction of breakup and coalescence times. Accurate knowledge of breakup and coalescence times in turn can directly impact the accuracy and usefulness of engineering analyses of breakup and coalescence phenomena utilized in population balance models. [41]

\section{ACKNOWLEDGMENTS}

This research was sponsored by the Petroleum Research Fund of the American Chemical Society, the Basic Energy Sciences Program of the United States Department of Energy, and the Engineering and Physical Sciences Research Council. All data accompanying this publication are available within the publication or from the corresponding author.

\section{Appendix A: Dynamically Growing Truncated Domain}

In this appendix, we describe and report results obtained with a variant of the truncated domain approach introduced in section IV. In contrast to the approach used in that section where $R_{T}$ is held fixed at a value equal to some multiple of the initial bridge radius (in the simulation results reported in that section, $R_{T}=100 R_{0}$ ), here the truncation radius is increased or moved dynamically so that the instantaneous value of $R_{T}(t)$ is some multiple of the instantaneous radius of the growing neck connecting the two bubbles. Figure 16 shows simulation results comparing predictions made using the new approach in which the truncation radius is dynamically varied as $R_{T}(t)=100 R_{\min }(t)$ to those in which results from different simulations each using a different fixed value of $R_{T}$ have been stitched together as described in section IV. This figure makes plain that simulation results obtained with the new and the old truncated domain approaches are in excellent agreement with one another.

\section{Appendix B: Accounting for the Fluid Dynamics of the Gas Bubbles}

In this short appendix, we investigate whether it is justified to neglect the dynamics of the flow within the bubbles and treating the gas within the bubbles as a dynamically passive fluid. To accomplish this goal, the flow

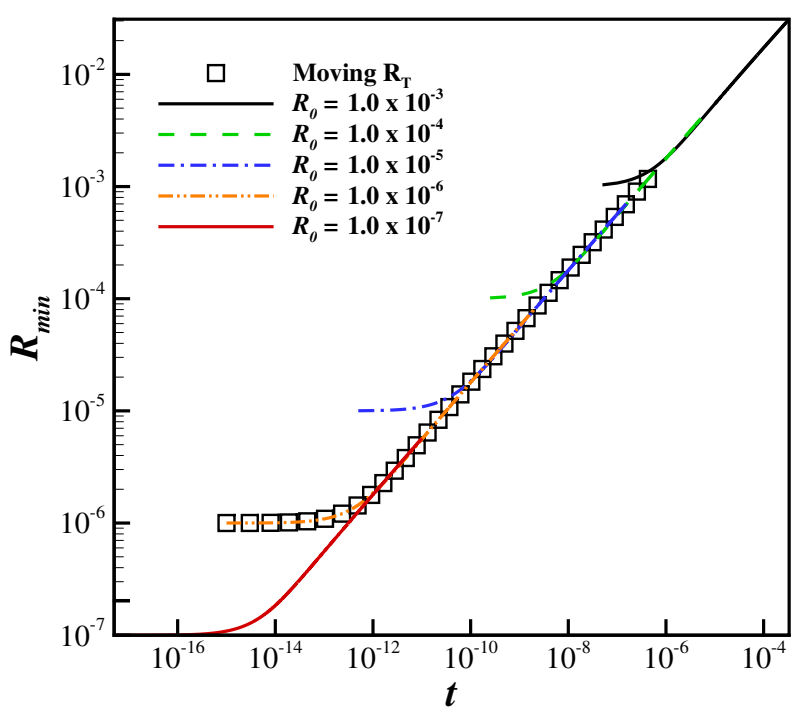

FIG. 16. Variation of the minimum radius $R_{\min }$ with time $t$ : comparison of simulation results obtained in a single computation with a truncated domain approach in which the truncation radius is varied dynamically as $R_{T}(t)=100 R_{\min }(t)$ (data points corresponding to the open symbols and labeled as Moving $R_{T}$ ) and those stitched together from different simulations each of which uses a fixed value of the truncation radius given by $R_{T}=100 R_{0}$ (curves each of which is identified by the value of the initial bridge radius used in a given simulation). In the simulation in which the truncation radius is varied continuously in time, $R_{0}=10^{-6}$. In all cases, $Z_{0}=R_{0}^{2}$ and $O h=3 \times 10^{-3}$.

within both the bubbles and surrounding liquid is determined together by extending the approach outlined earlier to simulate just the flow within the liquid exterior to the bubbles. For the details of solving the two-phase flow problem, the reader is referred to an earlier paper in which the finite element-based method that is described in this paper was applied to analyze the dynamics of two-fluid compound jets [42]. For additional details, the reader is also referred to the $\mathrm{PhD}$ thesis of Anthony [43].

When the dynamics of the fluid within the bubbles is accounted for, the problem is governed by three dimensionless groups: the Ohnesorge number $O h$ used earlier which is based on the properties of the outer liquid, the density ratio $D=\rho_{i} / \rho$, and the viscosity ratio $M=\mu_{i} / \mu$, where $\rho_{i}$ and $\mu_{i}$ denote the density and viscosity of the bubble fluid. Since the inner fluid in these simulations is a gas whereas the outer fluid is a liquid, both $D$ and $M$ will have values much less than one. For completeness, we will report results for two cases: in the first case, the liquid is a low-viscosity fluid and in the other, the liquid will be a high-viscosity fluid. However, the density ratio is kept fixed in the two simulations. The values of the dimensional parameters in these simulations are such that they are similar to those in select experiments performed 


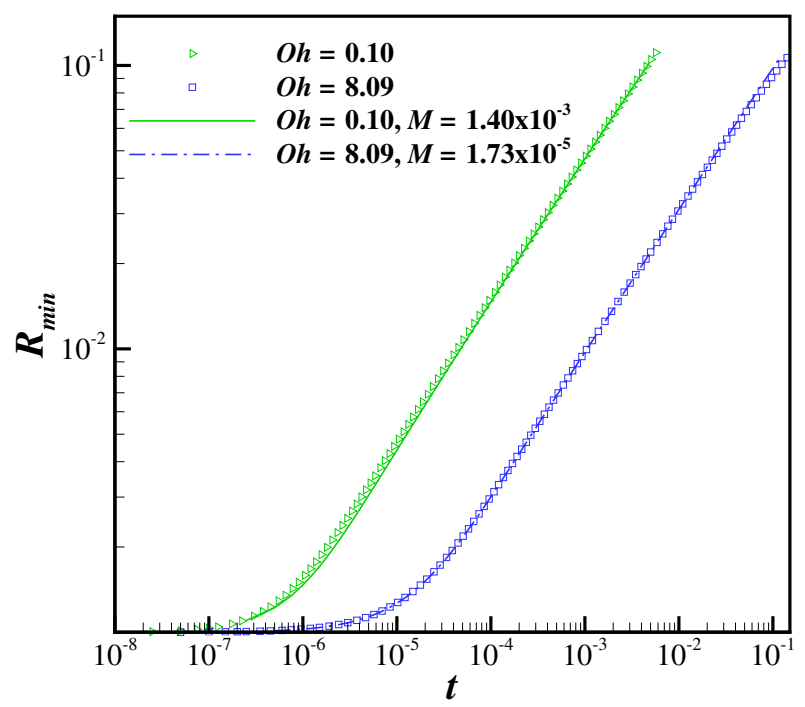

FIG. 17. Variation of the minimum radius $R_{\text {min }}$ with time $t$ : comparison of the radial scaling predicted by simulations in which the bubbles are treated as voids (the data points) and those in which the flow within the bubbles is determined along with the flow exterior to them (the curves). In all cases, $R_{0}=10^{-3}$ and $Z_{0}=R_{0}^{2}$. In the two-fluid simulations, $D=$ $1.48 \times 10^{-3}$.

by Paulsen et al. [25].

In Fig. 17, the variation of the minimum radius with time from four simulations is shown. In two of the sim- ulations, $O h=0.1$ and the other two, $O h=8.09$. For the same value of $O h$, in one of the simulations the bubbles are treated as passive voids and in the other the flow within the bubble is determined along with the flow in the exterior liquid. The results shown in Fig. 17 make plain that the variation of $R_{\min }$ with $t$ in both the lowviscosity and the high-viscosity limits is virtually identical whether the bubbles are treated as voids or the dynamics within them is accounted for. Thus, the neglect of the flow within the bubbles and treating them as passive voids, as had been the case throughout this paper, is an excellent approximation that remains true to the physics.

The responses depicted in Fig. 17 are consistent with the experimental findings of Paulsen et al. [25]. These authors have argued that while all coalescence events begin their lives in the inertially limited viscous (ILV) regime [17] dominated by the inner fluid, this regime would occur in such early times during bubble coalescence that it would be virtually impossible to observe in experiments using optical methods and, similarly, would be beyond the capability of computational methods as it would only exist for values of the minimum neck radius much smaller than the ones reported in Fig. 17. Thus, for the situations in Fig. 17 where $O h=0.1$, the transition from the ILV regime to the outer inertial regime shown in the figure would occur when $R_{\text {min }}=$ $O(O h M)=O\left(10^{-4}\right)$. Similarly, for the situations where $O h=8.09$, the transition from the ILV regime to the outer viscous regime shown in the figure would occur when $R_{\text {min }}=O(M)=O\left(10^{-5}\right)$.
[1] D. C. Blanchard and A. H. Woodcock, "Bubble formation and modification in the sea and its meteorological significance," Tellus 9, 145-158 (1957).

[2] E. A. Cassell, K. M. Kaufman, and E. Matijević, "The effects of bubble size on microflotation," Water Research 9, 1017 - 1024 (1975).

[3] C. A. Laamanen, G. M. Ross, and J. A. Scott, "Flotation harvesting of microalgae," Renewable and Sustainable Energy Reviews 58, 75 - 86 (2016).

[4] U. C. Bandara and P. D. Yapa, "Bubble sizes, breakup, and coalescence in deepwater gas/oil plumes," Journal of Hydraulic Engineering 137, 729-738 (2011).

[5] R. W. Hopper, "Coalescence of two equal cylinders: Exact results for creeping viscous plane flow driven by capillarity," Journal of the American Ceramic Society 67, C-262 (1984).

[6] R. W. Hopper, "Plane stokes flow driven by capillarity on a free surface," Journal of Fluid Mechanics 213, 349-375 (1990).

[7] J. Eggers, J. R. Lister, and H. A. Stone, "Coalescence of liquid drops," Journal of Fluid Mechanics 401, 293-310 (1999).

[8] A. Menchaca-Rocha, A. Martínez-Dávalos, R. Núñez, S. Popinet, and S. Zaleski, "Coalescence of liquid drops by surface tension," Physical Review E 63, 046309 (2001).

[9] L. Duchemin, J. Eggers, and C. Josserand, "Inviscid coalescence of drops," Journal of Fluid Mechanics 487, 167-178 (2003).

[10] M. Wu, T. Cubaud, and C. Ho, "Scaling law in liquid drop coalescence driven by surface tension," Physics of Fluids 16, L51-L54 (2004).

[11] S. T. Thoroddsen, T. G. Etoh, and K. Takehara, "The coalescence speed of a pendent and a sessile drop," Journal of Fluid Mechanics 527, 85-114 (2005).

[12] S. C. Case and S. R. Nagel, "Coalescence in low-viscosity liquids," Physical Review Letters 100, 084503 (2008).

[13] K. Fezzaa and Y. Wang, "Ultrafast x-ray phase-contrast imaging of the initial coalescence phase of two water droplets," Physical Review Letters 100, 104501 (2008).

[14] J. D. Paulsen, J. C. Burton, and S. R. Nagel, "Viscous to inertial crossover in liquid drop coalescence," Physical Review Letters 106, 114501 (2011).

[15] J. D. Paulsen, J. C. Burton, S. R. Nagel, S. Appathuri, M. T. Harris, and O. A. Basaran, "The inexorable resistance of inertia determines the initial regime of drop coalescence," Proceedings of the National Academy of Sciences 109, 6857-6861 (2012). 
[16] J. E. Sprittles and Y. D. Shikhmurzaev, "Coalescence of liquid drops: Different models versus experiment," Physics of Fluids 24, 122105 (2012).

[17] J. D. Paulsen, "Approach and coalescence of liquid drops in air," Physical Review E 88, 063010 (2013).

[18] L. Baroudi, M. Kawaji, and T. Lee, "Effects of initial conditions on the simulation of inertial coalescence of two drops," Computers and Mathematics with Applications 67, $282-289$ (2014).

[19] G. E. Charles and S. G. Mason, "The coalescence of liquid drops with flat liquid/liquid interfaces," Journal of Colloid Science 15, 236 - 267 (1960).

[20] D. G. A. L. Aarts and H. N. W. Lekkerkerker, "Droplet coalescence: drainage, film rupture and neck growth in ultralow interfacial tension systems," Journal of Fluid Mechanics 606, 275-294 (2008).

[21] W. Yao, H. J. Maris, P. Pennington, and G. M. Seidel, "Coalescence of viscous liquid drops," Physical Review E 71, 016309 (2005).

[22] T. Gilet, K. Mulleners, J. P. Lecomte, N. Vandewalle, and S. Dorbolo, "Critical parameters for the partial coalescence of a droplet," Physical Review E 75, 036303 (2007).

[23] H. Aryafar and H. P. Kavehpour, "Hydrodynamic instabilities of viscous coalescing droplets," Physical Review E 78, 037302 (2008).

[24] M. Yokota and K. Okumura, "Dimensional crossover in the coalescence dynamics of viscous drops confined in between two plates," Proceedings of the National Academy of Sciences 108, 6395-6398 (2011).

[25] J. D. Paulsen, R. Carmigniani, A. Kannan, J. C. Burton, and S. R. Nagel, "Coalescence of bubbles and drops in an outer fluid," Nature Communications 5, 3182 (2014).

[26] J. P. Munro, C. R. Anthony, O. A. Basaran, and J. R. Lister, "Thin-sheet flow between coalescing bubbles," Journal of Fluid Mechanics 773, R3 (2015).

[27] J. Q. Feng and O. A. Basaran, "Shear flow over a translationally symmetric cylindrical bubble pinned on a slot in a plane wall," Journal of Fluid Mechanics 275, 351-378 (1994).

[28] E. D. Wilkes, S. D. Phillips, and O. A. Basaran, "Computational and experimental analysis of dynamics of drop formation," Physics of Fluids 11, 3577-3598 (1999).

[29] K. N. Christodoulou and L. E. Scriven, "Discretization of free surface flows and other moving boundary problems,"
Journal of Computational Physics 99, 39 - 55 (1992).

[30] P. K. Notz and O. A. Basaran, "Dynamics and breakup of a contracting liquid filament," Journal of Fluid Mechanics 512, 223-256 (2004).

[31] Y. C. Liao, O. A. Basaran, and E. I. Franses, "Effects of dynamic surface tension and fluid flow on the oscillations of a supported bubble," Colloids and Surfaces A: Physicochemical and Engineering Aspects 282 - 283, 183 - 202 (2006).

[32] P. P. Bhat, O. A. Basaran, and M. Pasquali, "Dynamics of viscoelastic liquid filaments: Low capillary number flows," Journal of Non-Newtonian Fluid Mechanics 150, $211-225$ (2008).

[33] R. T. Collins, K. Sambath, M. T. Harris, and O. A. Basaran, "Universal scaling laws for the disintegration of electrified drops," Proceedings of the National Academy of Sciences 110, 4905-4910 (2013).

[34] P. M. Gresho and R. L. Sani, Incompressible Flow and the Finite Element Method (John Wiley \& Sons, 2000).

[35] P. Hood, "Frontal solution program for unsymmetric matrices," International Journal for Numerical Methods in Engineering 10, 379-399 (1976).

[36] G. I. Taylor, "The dynamics of thin sheets of fluid. III. disintegration of fluid sheets," Proceedings of the Royal Society of London A: Mathematical, Physical and Engineering Sciences 253, 313-321 (1959).

[37] F. E. C. Culick, "Comments on a ruptured soap film," Journal of Applied Physics 31, 1128-1129 (1960).

[38] J. B. Keller, "Breaking of liquid films and threads," Physics of Fluids 26, 3451-3453 (1983).

[39] R. M. S. M. Schulkes, "The contraction of liquid filaments," Journal of Fluid Mechanics 309, 277-300 (1996).

[40] Jens Eggers, "Nonlinear dynamics and breakup of freesurface flows," Review of Modern Physics 69, 865-930 (1997).

[41] T. Tobin, R. Muralidhar, H. Wright, and D. Ramkrishna, "Determination of coalescence frequencies in liquid-liquid dispersions: effect of drop size dependence," Chemical Engineering Science 45, 3491 - 3504 (1990).

[42] R. Suryo, P. Doshi, and O. A. Basaran, "Nonlinear dynamics and breakup of compound jets," Physics of Fluids 18, 082107 (2006).

[43] C. R. Anthony, Dynamics of Retracting Films and Filaments Near Singularities, Ph.D. thesis, Purdue University (2017). 